\title{
Serial Prefrontal Pathways Are Positioned to Balance Cognition and Emotion in Primates
}

\author{
Mary Kate P. Joyce, ${ }^{1,2}$ Miguel Ángel García-Cabezas, ${ }^{1,3}{ }^{\odot}$ Yohan J. John, ${ }^{1}$ and ${ }^{\circledR}$ Helen Barbas ${ }^{1}$ \\ ${ }^{1}$ Neural Systems Laboratory, Department of Health Sciences, Boston University, Boston, Massachusetts 02215, ${ }^{2}$ Graduate Program in Neuroscience, \\ Boston University School of Medicine, Boston, Massachusetts 02215, and ${ }^{3}$ Department of Anatomy, Histology, and Neuroscience, School of \\ Medicine, Universidad Autónoma de Madrid, Madrid, Spain 28029
}

The delicate balance among primate prefrontal networks is necessary for homeostasis and behavioral flexibility. Dorsolateral prefrontal cortex (dIPFC) is associated with cognition, while the most ventromedial subgenual cingulate area 25 (A25) is associated with emotion and emotional expression. Yet A25 is weakly connected with dlPFC, and it is unknown how the two regions communicate. In rhesus monkeys of both sexes, we investigated how these functionally distinct areas may interact through pregenual anterior cingulate area 32 (A32), which is strongly connected with both. We found that dlPFC innervated the deep layers of A32, while A32 innervated all layers of A25, mostly targeting spines of excitatory neurons. Approximately $20 \%$ of A32 terminations formed synapses on inhibitory neurons in A25, notably the powerful parvalbumin inhibitory neurons in the deep layers, and the disinhibitory calretinin neurons in the superficial layers. By innervating distinct inhibitory microenvironments in laminar compartments, A32 is positioned to tune activity in columns of A25. The circuitry of the sequential pathway indicates that when dIPFC is engaged, A32 can dampen A25 output through the parvalbumin inhibitory microsystem in the deep layers of A25. A32 thus may flexibly recruit or reduce activity in A25 to maintain emotional equilibrium, a process that is disrupted in depression. Moreover, pyramidal neurons in A25 had a heightened density of NMDARs, which are the targets of novel rapid-acting antidepressants. Pharmacologic antagonism of NMDARs in patients with depression may reduce excitability in A25, mimicking the effects of the neurotypical serial pathway identified here.

Key words: anterior cingulate; depression; dlPFC; inhibitory neurons; laminar connections; subgenual cingulate

\section{Significance Statement}

The anterior cingulate is a critical hub in prefrontal networks through connections with functionally distinct areas. Dorsolateral and polar prefrontal areas that are associated with complex cognition are connected with the anterior cingulate in a pattern that allows them to indirectly control downstream activity from the anterior cingulate to the subgenual cingulate, which is associated with heightened activity and negative affect in depression. This set of pathways provides a circuit mechanism for emotional regulation, with the anterior cingulate playing a balancing role for integration of cognitive and emotional processes. Disruption of these pathways may perturb network function and the ability to regulate cognitive and affective processes based on context.

\section{Introduction}

The functional and structural integrity of the prefrontal subgenual cingulate area 25 (A25) is necessary for emotional equilibrium. Emotional regulation also requires participation from

Received Apr. 12, 2020; revised July 18, 2020; accepted Aug. 15, 2020.

Author contributions: M.K.P.J. and H.B. designed research; M.K.P.J. and M.A.G.-C. performed research; M.K.P.J. and Y.J.J. analyzed data; M.K.P.J. wrote the first draft of the paper; M.K.P.J., M.A.G.-C., Y.J.J., and H.B. edited the paper; M.K.P.J. and H.B. wrote the paper.

This work was supported by National Institute of Mental Health R01MH117785 and R01MH057414, and National Institute of Neurological Disorders and Stroke R01NS024760, National Institutes of Health. We thank Marcia Feinberg and Jess Holz for electron microscopy expertise.

The authors declare no competing financial interests.

Correspondence should be addressed to Helen Barbas at barbas@bu.edu.

https://doi.org/10.1523/JNEUROSCI.0860-20.2020

Copyright $\odot 2020$ the authors prefrontal areas associated with cognition, such as area 10 (A10) and the dlPFC (Ramirez-Mahaluf et al., 2018). However, A25 has only sparse connections with the dIPFC and only a moderate connection with A10 (Joyce and Barbas, 2018). The pregenual anterior cingulate A32 is robustly connected with both A25 and the dlPFC (Barbas and Pandya, 1989; Barbas et al., 1999; Joyce and Barbas, 2018) and is associated with both affective and cognitive networks (Bush et al., 2000; Wang et al., 2016). A32 may thus act as an intermediate node allowing synergy between areas associated with cognition and emotion.

Recent and classical animal studies have demonstrated that A25 activity is associated with vagal tone, the stress response, and anhedonic behaviors (for review, see Alexander et al., 2019a). In humans, hyperactivity of the subgenual region is a biomarker for depression that is normalized in successful 
remission (Mayberg et al., 2005), a powerful demonstration that regulation of subgenual activity within prefrontal networks may be necessary for maintaining emotional flexibility.

A balance between dIPFC and A25 activation is necessary for the flexible response to stress (Datta and Arnsten, 2019). Hypoactivity in the dlPFC accompanies subgenual hyperactivity in clinical depression (Mayberg, 2003), and repetitive transcranial magnetic stimulation of the dlPFC brings remission to some patients (for review, see Gaynes et al., 2014; Trevizol and Blumberger, 2019). This evidence suggests that a dysregulated relationship between subgenual and dorsolateral cortices may accompany persistent mood disruption. Further, evidence that baseline activity in the human pregenual anterior cingulate predicts remittance in patients with depression (for review, see Waters and Mayberg, 2017), supports the idea that the pregenual cingulate plays a critical role in balancing prefrontal networks associated with emotional equilibrium.

The circuit mechanism underlying the inverse functional pathology between the dlPFC and the subgenual region in depression is not well understood. An important and frequently overlooked aspect of neural pathways is how they interface with excitatory and distinct types of inhibitory neurons that can regulate activity. In primates, a useful classification of inhibitory neurons can be performed by labeling for the calcium binding proteins (CBPs) parvalbumin (PV), calbindin (CB), and calretinin (CR), which label largely nonoverlapping populations of functionally distinct neurons that populate the cortical layers differentially (DeFelipe, 1997; for review, see Barbas et al., 2018). Our goal was to address how A32 axon terminations, arising from layers that receive input from A10 and the dlPFC, integrate with A25 microcircuitry, and especially with local inhibitory neurons in $\mathrm{A} 25$.

Beyond its inputs, another factor that affects cortical activity in A25 is the distribution and type of excitatory receptors on local neurons. For example, the density of NMDARs is especially high in human A25 (Palomero-Gallagher et al., 2008, 2009). This feature is of special interest in view of recent findings that NMDAR antagonists are an emerging therapeutic target for depression in humans and animal models (Fuchikami et al., 2015; Reinstatler and Youssef, 2015; for review, see Gould et al., 2019). Whether the antidepressant effects of NMDAR antagonists are mediated by populations of NMDARs on excitatory or inhibitory neurons in A25 remains an open question (for review, see Gould et al., 2019). We thus addressed whether NMDARs are preferentially distributed on pyramidal and functionally distinct inhibitory neurons in A25.

This study integrates circuit information at the pathway, laminar, cellular, and synaptic level to reveal how sequential cortical pathways originating in dlPFC may regulate the output of A25 through A32 as an intermediary. The circuitry illustrates that A32 can affect the balance between excitation and inhibition in A25 and may mediate the interaction between areas associated with cognition and emotion in prefrontal networks.

\section{Materials and Methods}

Experimental design. Figure 1 provides an overview of our experimental design. To study this serial cortical pathway connecting the dlPFC and A25, we injected bidirectional neural tracers into A32 (see Fig. $1 A$ ) of the rhesus macaque and used immunohistochemistry to visualize the tracers for optical and electron microscopy (EM). To map the pathways to/from the A32 injection sites, we used brightfield microscopy (BM) coupled with a commercial system that allowed for exhaustive plotting of neurons in the dlPFC and A10 that project to the A32 injection sites (see Fig. 1B). Neurons were grouped according to their laminar distribution (layers II-III or V-VI). We delineated the borders of prefrontal areas using matched coronal sections stained with Nissl paired with established architectonic criteria (Barbas and Pandya, 1989). We then used the same injection sites to stereologically estimate the density of A32 axon terminations, or boutons, in A25 (see Fig. 1C) according to their laminar distribution (layers I-III, V-VI).

Using BM and tissue-labeled immunohistochemically for the CBPs, we quantified the density of PV-, CB-, and CR-labeled neurons in A25, A32, and A46 by laminar group. Together, this provided a macro-level system overview, linking projection neurons in A10 and the dlPFC that project to A32 with A32 neurons that sent axonal terminations to distinct microenvironments of A25. In summary, we characterized the serial projections and laminar microsystems that may enable signals to flow from A10 and the dlPFC ultimately to A25.

We then used high-resolution confocal fluorescence microscopy (CM) and EM to study specific interactions of A32 terminations with excitatory and inhibitory postsynaptic targets in A25 (see Fig. 1D). We used multiple immunohistochemical labeling to visualize neuronal tracers with $\mathrm{PV}, \mathrm{CB}$, or CR. Using stacks obtained by $\mathrm{CM}$, we analyzed appositions of tracer-labeled axonal terminations with dendrites labeled for PV, CB, or CR in superficial and deep layers. Because PV, CB, and CR label the overwhelming majority of inhibitory neurons in the cortex, by subtraction we could compute the proportion of axonal terminations presumed to appose excitatory structures. This produced a meso-level portrait of the putative targets of A32 terminations in A25 with a large sample size. We then used EM to examine these proportions at the micro- or synapse-level, where long series of ultrathin sections allowed for $3 \mathrm{D}$ reconstruction to examine structural features of postsynaptic targets. We analyzed data and performed statistical analyses using SPSS, as described below.

Surgery, tracer injection, and perfusion. Table 1 lists the cases, tracers, and analyses performed. Experiments were conducted on 15 rhesus monkeys (Macaca mulatta, 2-5.5 years, $n=8$ female) according to protocols approved by the Institutional Animal Care and Use Committee at Boston University School of Medicine, Harvard Medical School, and New England Primate Research Center, in compliance with the National Institutes of Health Guide for the care and use of laboratory animals (publication 80-22 revised, 1996, Office of Science and Health Reports, Division of Receipt and Referral/ National Institutes of Health). Procedures were designed to minimize animal suffering. In each case, injections of multiple distinct tracers were placed to address questions in this and other unrelated studies, thus minimizing the number of animals needed for research (Barbas, 1993; Dombrowski and Barbas, 1996; Zikopoulos and Barbas, 2006; Ghashghaei et al., 2007; García-Cabezas and Barbas, 2017; Garcia-Cabezas et al., 2017).

For tracer injections, we conducted MRI on each animal after sedation (ketamine hydrochloride, $10-15 \mathrm{mg} / \mathrm{kg}$, i.m.), anesthesia (propofol, loading dose $2.5-5 \mathrm{mg} / \mathrm{kg}$, i.v., continuous infusion rate $0.25-0.4 \mathrm{mg} / \mathrm{kg} /$ $\mathrm{min})$, and placement in a stereotaxic apparatus (1430 M, David Kopf Instruments). MRI scans were registered to the stereotaxic space and used to calculate stereotaxic coordinates for injection of neural tracers.

About a week later, animals were first sedated and then placed under general anesthesia (isoflurane, to a surgical level). We then performed surgery under sterile conditions with continuous monitoring of respiratory rate, oxygen saturation, heart rate, and temperature. Each animal's head was placed in the stereotax to match its original MRI placement. A microdrive attachment was used to mount syringes for precise localization of planned injection coordinates. After exposing a small area of cortex above designated injection sites, microsyringes (Hamilton, 5 or $10 \mu \mathrm{l}$ ) were loaded with tracer (biotinylated dextran amine [BDA] and Lucifer yellow [LY]; Table 1), mounted, and guided toward the final injection site coordinates. For both tracers (BDA, LY), we mixed molecular weight variants to produce bidirectional tracing, as the $3 \mathrm{kDA}$ dextran amine variant is optimal for labeling of neuronal cell bodies and proximal dendrites, while the $10 \mathrm{kDA}$ dextran amine variant is optimal for labeling axonal terminations (Veenman et al., 1992; Richmond et al., 1994; Reiner et al., 2000). To avoid tracer leakage during insertion, we loaded syringes with a small bubble of air after aspiration of the tracer. Syringes 


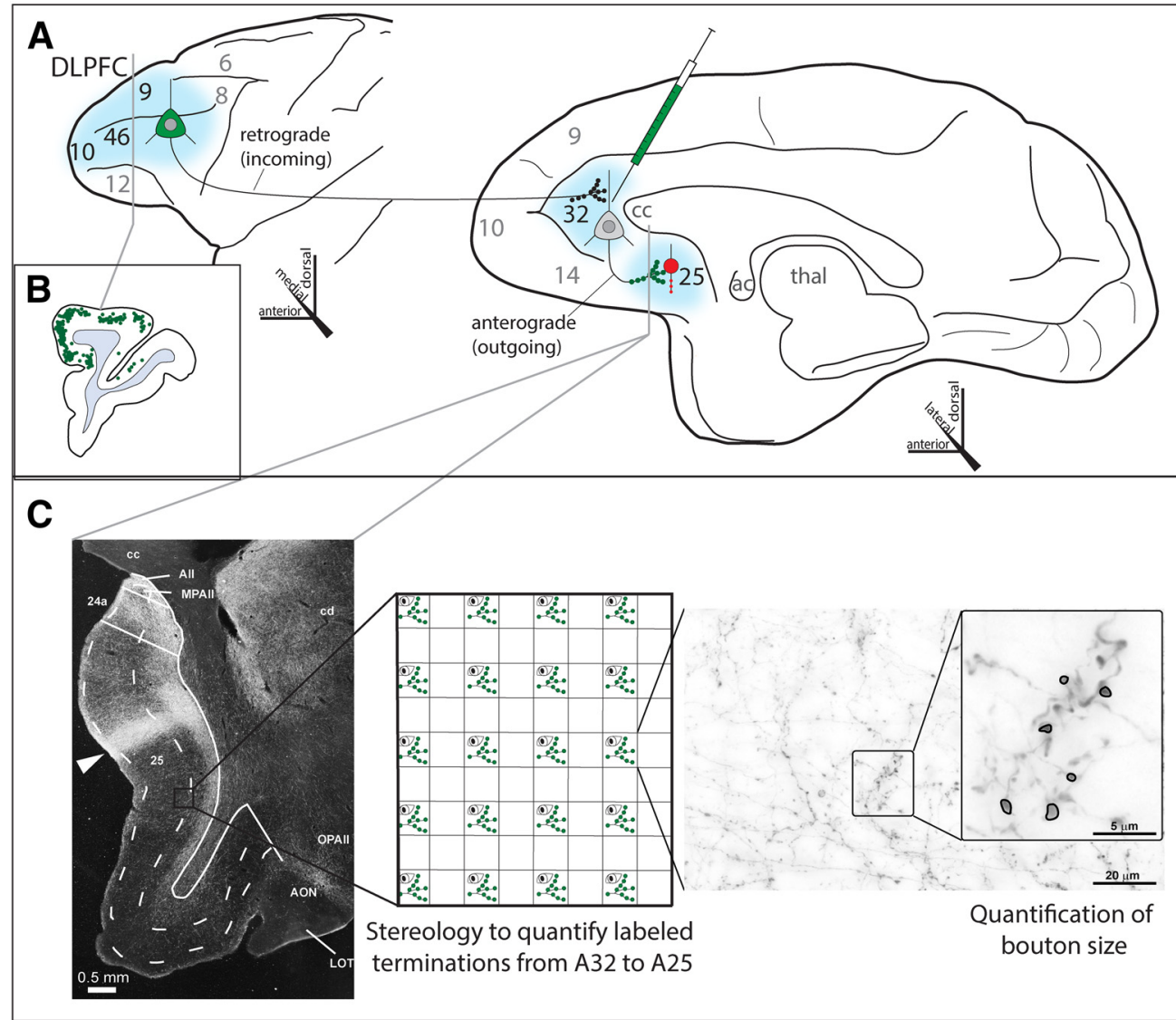

\section{System}

D
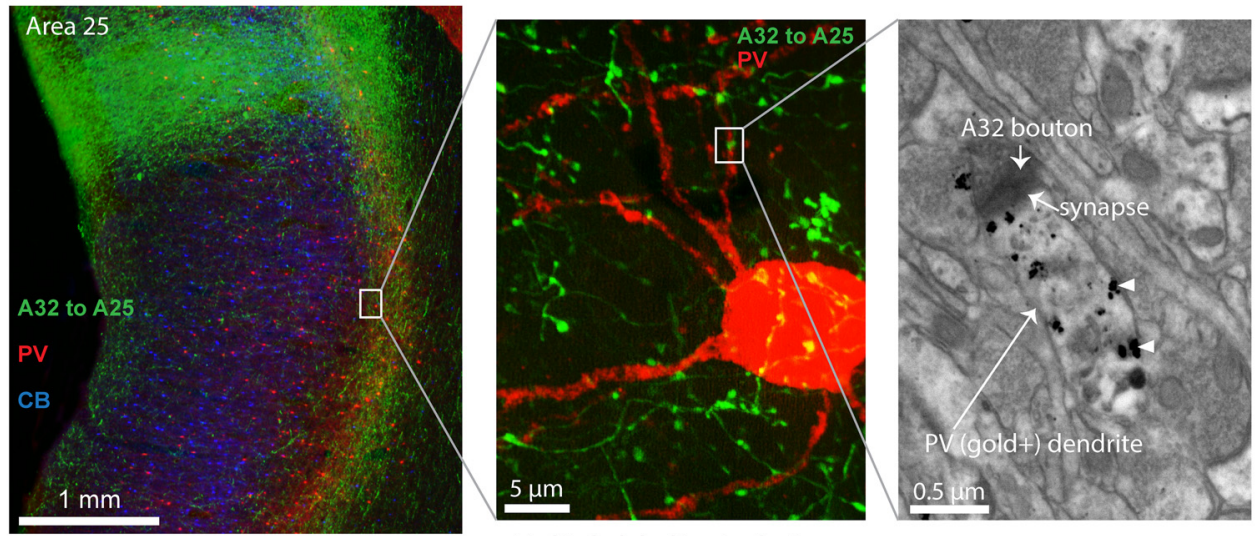

Multiple labeling techniques

to examine postsynaptic targets

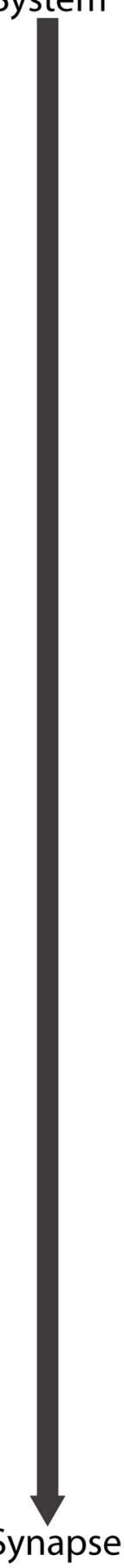

Figure 1. Experimental design. $\boldsymbol{A}$, Schematic represents the serial pathway under study on the lateral (left) and medial (right) surfaces of the rhesus monkey brain. A bidirectional tracer injected into A32 retrogradely labeled neurons in the dIPFC/A10 and anterogradely labeled axonal terminations in A25 to produce a sequential set of labeled pathways. $\boldsymbol{B}$, Example plot of a prefrontal coronal section exhaustively sampled to map retrogradely labeled neurons (green). C, Schematic represents the sampling of axonal terminations for density computation and quantification of bouton size. Darkfield photomicrograph (left) of a coronal slice through A25. BDA from an A32 injection appears bright, forming a columnar patch (white arrowhead) just above the rostral sulcus. Dotted lines indicate the bottom of layer I and the top of layer V. Middle, A simplified version of the optical fractionator method of stereological sampling, where a grid is placed over the ROI and fields are sampled at a rate that minimizes a coefficient of error. Brightfield photomicrographs (right) were taken at different focal planes through the depth of tissue, and the boutons were measured for major diameter. $\boldsymbol{D}$, Series of photomicrographs represent the analysis of the postsynaptic targets of A32 terminations in A25. Left, Coronal section through A25 at the rostral sulcus. BDA (green) forms a columnar patch near the top of the image. CB neurons (blue) are more prevalent in the superficial layers, and PV neurons (red) are most prevalent in the middle to deep layers. Middle, Maximum projection of high magnification z stack (CM) in the deep layers of A25 represents A32 (green) axonal terminations in some cases closely apposing PV neuronal processes (red). Right, Electron photomicrograph represents the end of an A32 bouton labeled with DAB (dark, black precipitate) forming a synapse on a dendrite that has abundant gold labeling for PV (deeply black clustered quanta, white arrowheads). Numbers indicate cortical areas according to Barbas and Pandya (1989). ac, Anterior commissure; All, allocortex; AON, anterior olfactory nucleus; cc, corpus callosum; cd, caudate; LOT, lateral olfactory tract; MPAll, medial periallocortex; OPAll, orbital periallocortex; ROI, region of interest; thal, thalamus. 
Table 1. List of cases and analysis performed ${ }^{a}$

\begin{tabular}{|c|c|c|c|c|}
\hline Case & Sex & Age (yr) & Tracer/injection site & Analysis \\
\hline AY-L & $\mathrm{F}$ & 3 & $\mathrm{BDA}, \mathrm{A} 32$ & System-level mapping \\
\hline BG-R & $\mathrm{F}$ & 2 & BDA, A32 & System-level mapping \\
\hline BI-R & $\mathrm{F}$ & 3 & BDA, A32 & System-level mapping, apposition analysis, and synaptic analysis \\
\hline BL-R & M & 3 & $L Y, A 32$ & System-level mapping \\
\hline BN-L & M & 2 & $L Y, A 32$ & System-level mapping \\
\hline BS-R & $\mathrm{F}$ & 3.5 & BDA, A32 & System-level mapping, apposition analysis, and synaptic analysis \\
\hline AS & $\mathrm{F}$ & - & NA & Stereology in $\mathrm{A} 46, \mathrm{~A} 25, \mathrm{~A} 32$ for PV, CB \\
\hline AT & $\mathrm{F}$ & - & NA & Stereology in A46, A25, A32 for PV, CB \\
\hline AV & - & - & NA & Stereology in A46, A25, A32 for PV, CB, CR, cytoarchitecture photography \\
\hline$A X$ & - & - & NA & Stereology in A46, A25, A32 for CR \\
\hline 695 & - & - & NA & Stereology in A46, A25, A32 for CR \\
\hline BU-R & M & 4 & NA & CB/GABA colocalization \\
\hline $\mathrm{BO}-\mathrm{R}$ & M & 3 & NA & GAD-67 for $C B / G A B A$ analysis \\
\hline BR-R & $\mathrm{F}$ & 3 & NA & NR1 and PV, CB, CR colocalization \\
\hline BW-R & $\mathrm{F}$ & 5.5 & NA & NR1 and PV, CB, CR colocalization \\
\hline
\end{tabular}

${ }^{a}$ NR1, Obligatory subunit of NMDAR. BDA, biotinylated dextran amine; CB, calbindin; CR, calretinin; GABA, gamma-amino-butyric acid; LY, Lucifer Yellow; NR1, obligatory subunit of N-methyl-D-aspartate receptor.

Table 2. List of antibodies and dilutions used ${ }^{a}$

\begin{tabular}{|c|c|c|c|c|c|}
\hline Antibody & RRID & Use & Dilution & Case & Colocalization \\
\hline Mouse anti-PV, Swant, catalog \#235 & $A B \_10000343$ & Primary & $1: 2000$ & $\mathrm{Bl}, \mathrm{BS}$ & $\begin{array}{l}\text { CM, } 32 \rightarrow 25 \text { appositions } \\
\quad \text { EM, } 32 \rightarrow 25 \text { synaptic analysis }\end{array}$ \\
\hline Mouse anti-CB, Swant, catalog \#300 & $A B \_10000347$ & Primary & $1: 2000$ & $\begin{array}{r}\mathrm{BI}, \mathrm{BS} \\
\mathrm{BU}\end{array}$ & $\begin{array}{l}\text { CM, } 32 \rightarrow 25 \text { appositions } \\
\quad \text { CM, CB/GABA colocalization }\end{array}$ \\
\hline Mouse anti-CR, Swant, catalog \#6B3 & AB_10000320 & Primary & $1: 2000$ & $\mathrm{Bl}, \mathrm{BS}$ & CM, $32 \rightarrow 25$ appositions \\
\hline Rabbit anti-PV, Swant, catalog \#pv27 & AB_2631173 & Primary & $1: 2000$ & $\mathrm{BR}$ & NR1/PV, CB, CR colocalization \\
\hline Rabbit anti-CB, Swant, catalog \#cb38 & $A B \_10000340$ & Primary & $1: 2000$ & $\mathrm{BR}$ & NR1/PV, CB, CR colocalization \\
\hline Rabbit anti-CR, Swant, catalog \#7697 & AB_2721226 & Primary & $1: 2000$ & $\mathrm{BR}$ & NR1/PV, CB, CR colocalization \\
\hline Rabbit anti-GABA, Immunostar, catalog \#20095 & $A B \_572233$ & Primary & $1: 1000$ & BU & $\mathrm{CM}, \mathrm{CB} / \mathrm{GABA}$ colocalization \\
\hline Mouse anti-GAD-67, Millipore, catalog \#MAB5406 & AB_2278725 & Primary & $1: 500$ & $\mathrm{BO}$ & CB/GABA colocalization \\
\hline Mouse anti-NR1, Millipore, catalog \#MAB363 & & Primary & $1: 250$ & $\mathrm{BR}$ & NR1/PV, CB, CR colocalization \\
\hline Rabbit anti-LY, Molecular Probes, catalog \#A-5750 & AB_2536190 & Primary & $1: 800$ & $\mathrm{BL}, \mathrm{BN}$ & BM system-level mapping \\
\hline AlexaFluor-405 Streptavidin, Thermo Fisher Scientific, catalog \#S-32351 & & Secondary & $1: 100$ & $\mathrm{Bl}, \mathrm{BS}$ & CM, $32 \rightarrow 25$ appositions \\
\hline AlexaFluor-488 Streptavidin, Thermo Fisher Scientific, catalog \#S-32354 & & Secondary & 1:100 & $\mathrm{Bl}, \mathrm{BS}$ & CM, $32 \rightarrow 25$ appositions \\
\hline Biotinylated goat anti-mouse, Vector, catalog \#BA-9200 & $A B \_2336171$ & Secondary & $1: 200$ & BU & $\mathrm{CB} / \mathrm{GABA}$ colocalization \\
\hline AlexaFluor-647 goat anti-mouse, Thermo Fisher Scientific, catalog \#A-28181 & & Secondary & $1: 100$ & $\mathrm{BI}, \mathrm{BS}$ & CM, $32 \rightarrow 25$ appositions \\
\hline AlexaFluor-568 goat anti-mouse, Thermo Fisher Scientific, catalog \#A-11031 & & Secondary & $1: 100$ & $\begin{array}{r}\mathrm{Bl}, \mathrm{BS} \\
\mathrm{BU}\end{array}$ & $\begin{array}{l}\text { CM, } 32 \rightarrow 25 \text { appositions } \\
\quad \text { CM, CB/GABA colocalization }\end{array}$ \\
\hline AlexaFluor-405 goat anti-mouse, Thermo Fisher Scientific, catalog \#A-31553 & & Secondary & 1:100 & $\mathrm{Bl}, \mathrm{BS}$ & $\mathrm{CM}, 32 \rightarrow 25$ appositions \\
\hline AlexaFluor-647 goat anti-rabbit, Thermo Fisher Scientific, catalog \#A-27040 & & Secondary & 1:100 & $\begin{array}{l}\mathrm{BU} \\
\mathrm{BR}\end{array}$ & $\begin{array}{l}\text { CM, CB/GABA colocalization } \\
\quad \text { NR1/PV, CB, CR colocalization }\end{array}$ \\
\hline Ultrasmall ImmunoGold F(ab) goat anti-mouse, Aurion, catalog \#800.366 & AB_2315632 & Secondary & $1: 100$ & $\mathrm{Bl}, \mathrm{BS}$ & EM, $32 \rightarrow 25$ synaptic analysis \\
\hline
\end{tabular}

${ }^{a}$ NR1, Obligatory subunit of NMDAR. BDA, biotinylated dextran amine; BM, brightfield microscopy; CB, calbindin; CM, confocal microscopy; CR, calretinin; EM, electron microscopy; PV, parvalbumin; GABA, gamma-amino-butyric acid; GAD-67, glutamate decarboxylase-67; LY, Lucifer yellow; NR1, obligatory subunit of N-methyl-D-aspartate receptor.

were left at the site of injection for 5-10 min to allow for local diffusion of the tracer, and to prevent backward suction of the tracer during syringe retraction. Animals were monitored postoperatively and given antibiotics and analgesics as needed.

After a tracer propagation period of 18-20 d, animals were sedated, given a lethal dose of anesthetic (sodium pentobarbital, to effect), and transcardially perfused ( $4 \%$ paraformaldehyde, $0.2 \%$ glutaraldehyde in 0.1 м PBS, pH 7.4; Cases AY, BG, BI, BL, BN, BO, BR, BS, BU, BW; 4\% PFA in 0.1 m cacodylate buffer, $\mathrm{pH}$ 7.4; Cases AS, AT, AV, AX, 695). Brains were removed, photographed, cryoprotected in ascending sucrose solutions ( $10 \%-25 \%$ sucrose, $0.05 \%$ sodium azide in $0.1 \mathrm{M} \mathrm{PB}, \mathrm{pH} 7.4$ ), frozen in $-80^{\circ} \mathrm{C}$ isopentane, and sectioned on a freezing microtome (AO Scientific Instruments/Reichert Technologies) at 40 or $50 \mu \mathrm{m}$. We collected sections systematically into matched series and placed them in antifreeze (30\% ethylene glycol, $30 \%$ glycerol, $0.05 \%$ sodium azide in $0.01 \mathrm{M} \mathrm{PB}, \mathrm{pH}$ 7.4) for long-term storage.

Labeling procedures for $B M, C M$, and $E M$. We processed free-floating sections with immunohistochemical techniques after first rinsing them of cryoprotectant using $\mathrm{PB}(0.1 \mathrm{M}, \mathrm{pH} 7.4)$. Antigen retrieval was performed in sodium citrate solution (10 mm, $\mathrm{pH} 8.5$, Sigma Millipore) using a $70^{\circ} \mathrm{C}-80^{\circ} \mathrm{C}$ water bath $(\mathrm{BM}, \mathrm{CM})$ or $30^{\circ} \mathrm{C}-35^{\circ} \mathrm{C}$ microwave protocol $(150$ W, $15 \mathrm{~min}$ with temperature monitoring, BioWave, Ted Pella; EM). Sections were rinsed and incubated in $0.05 \mathrm{~m}$ glycine $\left(4^{\circ} \mathrm{C}, 1 \mathrm{~h}\right.$, Sigma Millipore) to bind free aldehydes (BM, CM, EM). Following rinsing, sections were incubated in $0.3 \% \mathrm{H}_{2} \mathrm{O}_{2}$ in $0.1 \mathrm{M} \mathrm{PB}(30 \mathrm{~min})$ to quench endogenous peroxidases (BM, EM). Sections were then rinsed and blocked in preblocking solution, which contained $10 \%$ normal serum of the secondary antibody host animal (Sigma disector Millipore; BM, CM, EM), 0.2\% BSA-c (Aurion; BM, CM, EM), Triton X-100 (0.2\% Sigma Millipore; BM, CM), or reduced Triton-X100 (0.025\%, Roche Applied Science; EM), and cold water fish gelatin $(0.1 \%$, Aurion; EM) for stabilization of ultrastructure.

Sections were incubated with primary antibodies (Table 2), for 1-3 d, rinsed, then incubated in secondary antibodies (Table 2) for $3 \mathrm{~h}$ to $1 \mathrm{~d}$. For control sections, primary antibodies were omitted. Incubation was enhanced by microwaving $\left(2 \mathrm{~min}\right.$ at $\left.150 \mathrm{~W}, 4^{\circ} \mathrm{C}\right)$. To visualize structures of interest after secondary antibody incubation for BM, sections were rinsed and incubated in avidin-biotin horseradish complex (ABC, 1:100 
in $0.1 \mathrm{M} \mathrm{PB}, \mathrm{AB}-\mathrm{HRP}$, catalog \#PK-6100, Vector, RRID:AB_2336827) and then processed with diaminobenzidine (DAB) for 1-3 min (catalog \#SK-4100, Vector, RRID:AB_2336382).

After completion of immunohistochemistry for BM, sections were mounted on gelatin-coated glass slides and dried. Nissl counterstaining (thionin stain) as previously described (García-Cabezas et al., 2016) was performed on every other section to aid in cytoarchitectonic parcellation. BM sections were coverslipped with Entellan (Sigma Millipore). For $\mathrm{CM}$, sections were mounted and coverslipped with Fluorosave (Calbiochem) and edges were hardened using fast-drying clear nail polish (Sally Hansen), which helps prevent the infiltration of air bubbles into the coverslipping medium.

For EM, after primary antibody incubation, structures of interest were incubated in a gold-conjugated secondary antibody (Table 2), which was followed by silver enhancement of gold particles ( $90 \mathrm{~min}$, R-GENT SE-EM kit 500.033, Electron Microscopy Sciences, catalog \#255213) and then quenched $0.1 \mathrm{M}$ PB rinses followed by enhancement solution (Enhancement conditioning solution $10 \times 500.055$, Electron Microscopy Sciences, catalog \#25830). Sections were postfixed using reduced glutaraldehyde fixative soqlution in the microwave, as above, and then incubated in secondary antibodies for visualization with $\mathrm{ABC}$ and $\mathrm{DAB}$, as above, to allow for accurate dissection of regions of interest (ROI) after heavy metal infiltration. Sections were photographed using a temporary $0.1 \mathrm{M} \mathrm{PB}$ mount on an unsubbed glass slide before commencing with the remainder of EM processing. We performed microwave postfixation $(6 \%$ glutaraldehyde, $2 \%$ PFA in $0.1 \mathrm{M}$ $\mathrm{PB}, 150 \mathrm{~W}, 15^{\circ} \mathrm{C}$ ) until sample temperatures reached $30^{\circ} \mathrm{C}-35^{\circ} \mathrm{C}$, then left the sections in the fixative to come to room temperature for $30 \mathrm{~min}$, which was followed by $0.1 \mathrm{M}$ PB rinses.

EM processing was conducted using either a routine protocol (Case BI) or a modified protocol optimized for high-throughput block-face imaging (Cases BI and BS). Routine EM methods for ultrathin sectioning have been described previously (Zikopoulos et al., 2016; García-Cabezas and Barbas, 2017). For the modified protocol, we postfixed tissue sections for $36 \mathrm{~min}$ in $2 \%$ osmium tetroxide (Electron Microscopy Sciences) with $1.5 \%$ potassium ferrocyanide in $0.1 \mathrm{M} \mathrm{PB}$ under vacuum with an initial microwave session $\left(100 \mathrm{~W}\right.$ at $\left.4^{\circ} \mathrm{C}, 6 \mathrm{~min}\right)$. After three $\mathrm{dH}_{2} \mathrm{O}$ rinses, we incubated the tissue sections for $30 \mathrm{~min}$ in $1 \%$ thiocarbohydrazide in $\mathrm{dH}_{2} \mathrm{O}$ (Sigma Millipore), followed by another three $\mathrm{dH}_{2} \mathrm{O}$ rinses. We then incubated tissue sections in a second osmium solution ( $2 \%$ osmium tetroxide in $\mathrm{dH}_{2} \mathrm{O}$ ), under vacuum, with an initial microwave session $\left(100 \mathrm{~W}\right.$ at $\left.4^{\circ} \mathrm{C}, 6 \mathrm{~min}\right)$, for a total of $36 \mathrm{~min}$. Sections were rinsed in three $\mathrm{dH}_{2} \mathrm{O}$ rinses, and stained overnight at $4^{\circ} \mathrm{C}$ in $1 \%$ uranyl acetate (Electron Microscopy Sciences) in $\mathrm{dH}_{2} \mathrm{O}$. The next day, we rinsed the sections with $\mathrm{dH}_{2} \mathrm{O} 3$ times, and then incubated them in lead aspartate solution $(0.066 \mathrm{~g}$ lead nitrate, Electron Microscopy Sciences, in $10 \mathrm{ml}$ of $0.4 \% \mathrm{~L}$-aspartic acid in $\mathrm{dH}_{2} \mathrm{O}$, titrated to $\mathrm{pH} 5.5$ using $20 \%$ potassium hydroxide solution, Sigma Millipore) for $30 \mathrm{~min}$ at $60^{\circ} \mathrm{C}$. We then dehydrated the sections in ascending graded ethanols (50\%, 75\%, 85\%, 95\%, $100 \%, 3 \times 5 \mathrm{~min}$ each). Sections were then infiltrated with propylene oxide $(2 \times 10 \mathrm{~min}$, Electron Microscopy Sciences), followed by a 1:1 mixture of LX112 resin (LX112 Embedding Kits, Ladd Research Industries) and propylene oxide for $1 \mathrm{~h}$, and then finally with a 2:1 mixture of $\mathrm{LX} 112$ and propylene oxide at $25^{\circ} \mathrm{C}$ overnight. The following day, the sections were infiltrated with pure LX112 resin for $4 \mathrm{~h}$ under vacuum, and then flat embedded in LX112 resin between sheets of Aclar (Ted Pella), and cured for at least $48 \mathrm{~h}$ at $60^{\circ} \mathrm{C}$.

Pathway mapping using BM: dlPFC and A10 inputs to A32; A32 terminations in A25. In 3 cases (AY, BG, BI) that had been processed to visualize BDA for $\mathrm{BM}$ with $\mathrm{DAB}$, we used exhaustive plotting to map pathways directed to $\mathrm{A} 32$ from $\mathrm{A} 9, \mathrm{~A} 10$, and $\mathrm{A} 46$ in a series of coronal sections at equal intervals ( 0.8 or $1 \mathrm{~mm}$, depending on section thickness) through the hemisphere ipsilateral to the injection site. In each series, we mapped all labeled cortical neurons at $200 \times$ using BM and a semiautomated commercial system (Neurolucida, RRID:SCR_001775; Olympus BX60). Labeled neurons were categorized into one of two classes: superficial (layers II-III) or deep (layers V-VI). To place architectonic borders, we used the Nissl-counterstained sections to compare architecture to the prefrontal map (Barbas and Pandya, 1989). The number of neurons in each laminar category of each area was summed across coronal sections and used to determine the laminar placement of neurons directed to A32 from these areas, expressed as a percent of neurons found in superficial layers. Percentages were averaged across cases and expressed with SEM.

In 5 cases ( $\mathrm{AY}, \mathrm{BG}, \mathrm{BI}, \mathrm{BL}, \mathrm{BN})$, we used unbiased stereological methods to quantify A32 terminations in A25 using series of 5-7 coronal sections separated by intervals of $1 \mathrm{~mm}$. In each sampled section, the entirety of the medial and orbital extent of A25 was sampled for stereological analysis. We performed stereology using the optical fractionator method via a semi-automated commercial system (StereoInvestigator, RRID:SCR_002526; Olympus BX60). The optical fractionator extrapolates a density for the ROI based on section thickness and systematic sampling at regular intervals (Howard and Reed, 1998). We used a disector of $(25 \mu \mathrm{m})^{2}$, a grid size of $(250 \mu \mathrm{m})^{2}$, and a disector height of $5-11 \mu \mathrm{m}$ with a guard zone of $2 \mu \mathrm{m}$ at the top and bottom of each section. This sampling rate yielded a coefficient of error $<10 \%$, as recommended (Gundersen, 1986). Boutons were counted at $1000 \times$ with oil immersion. We computed the density of terminations by dividing the estimated number of terminations by the estimated volume of each region separately for laminar groups and all layers together. We expressed laminar specificity as the ratio of the density in deep layers to the density in all layers. The ratio was averaged across cases and expressed with SEM. We exported a plot of each section counted for stereological estimation of bouton density to generate a map of each injection site's range of innervation in A25 based on our sampling of 5-7 sections for stereology. Using the sampled sites on each section, we assigned an index based on density of terminations, and those indices were used to populate A25 in a normalized brain space. These schematics show a map of each injection site's distribution of terminations in A25, and their overlap across A32 injection sites.

In 3 of these cases (AY, BG, BI), we measured the major diameter of A32 axonal diameters in laminar groups of A25. In layers I, II-III, and $\mathrm{V}$-VI, we obtained BM $z$ stacks with a step size of $0.5 \mu \mathrm{m}$. Stacks were imported into the software program Reconstruct (SynapseWeb; RRID: SCR_002716; Fiala, 2005) to measure the major diameter of all A32 terminations.

To demonstrate the distinct morphologic and labeling properties of $\mathrm{CB}^{+}$inhibitory and presumed excitatory pyramidal neurons, we doublelabeled for GABA and $\mathrm{CB}$ for $\mathrm{CM}$ (Case $\mathrm{BU}$ ), and compared the results with the BM tissue labeled for $\mathrm{CB}$ or decarboxylase-67 (GAD-67), a marker of inhibitory neurons. This analysis allowed us to segregate the $\mathrm{CB}^{+}$labeled neurons in A25, A32, and A46 into two separate populations of darkly labeled, often multipolar, presumed inhibitory neurons, or lightly labeled, pyramidal-shaped, presumed excitatory neurons with an unlabeled nucleus. To quantify the density of PV, CR, and CB (presumed excitatory or inhibitory) neurons, we used stereological sampling, as above. Sampling was performed in representative columns in the gyral parts of A32 and A25, and the dorsal limb of caudal dorsolateral 46, using parameters previously described (Garcia-Cabezas et al., 2017). We computed neuron density for laminar groups and for all layers together by dividing the estimated number of counted cells by the estimated volume.

Appositional analysis of A32 in A25: CM. After A25 sections were processed for immunofluorescence of the tracer (BDA, Cases BI, BS) and $\mathrm{PV}, \mathrm{CB}$, or $\mathrm{CR}$, they were imaged using a laser-scanning confocal microscope (Axio Observer Z1, LSM 880, Carl Zeiss) at 630× (Zen 2.1 package, RRID:SCR_013672). Green fluorophores were imaged using a $488 \mathrm{~nm}$ argon ion laser, red fluorophores with a 568 DPSS 561-10 laser, blue fluorophores with a Diode 405-30 laser, and far-red fluorophores were imaged with a $633 \mathrm{~nm}$ helium neon laser (all Carl Zeiss). We acquired stacks of optical sections $(0.31$ or $0.33 \mu \mathrm{m}$ step size). Stacks were deconvolved (Huygens Professional 17.10, Scientific Volume Imaging) to minimize the effect of the point spread function. We exhaustively sampled all A32 terminations within each stack and determined the proportion apposed to neuronal elements immunolabeled for PV, CB, or CR. Appositions were defined as a close contact between the tracer and a CBP-labeled structure, often visible by a point of colocalization. The proportion apposed to presumed excitatory structures was computed using subtraction.

Synaptic analysis of A32 in A25: EM. A25 sections were immunolabeled for EM synaptic analysis (Cases BI, BS). BDA was labeled using 
$\mathrm{DAB}$, which forms a dark precipitate that fills the cytoplasm of neuronal elements containing tracer. PV, CB, or CR was labeled using gold, which forms small quanta that sometimes cluster. After immunolabeling, we followed the above protocol for impregnation of heavy metals for EM. Then, using a stereoscope, we dissected small cubes of Aclar-embedded tissue in the superficial and mid-to-deep layers of A25, using the photographs of each section taken before EM processing to identify fiduciary landmarks (e.g., blood vessels) and precisely locate our regions of interest. The small cubes of tissue were placed on top of premade LX112 resin blocks in a drop of fresh resin. These blocks were then cured for $\geq 48 \mathrm{~h}$ at $60^{\circ} \mathrm{C}$. For block-face imaging, cubes of tissue were affixed to aluminum pins using conductive epoxy glue (Chemtronics, catalog \#CW2400). We used an ultramicrotome (Ultracut UCT, Leica Microsystems) to expose the surface of the tissue in both the resin blocks or pins. After exposing the tissue, pins for block-imaging were painted with conductive silver paint (Ted Pella, catalog \#16035), which reduces charging artifacts in the scanning electron microscope (SEM). After the tissue was exposed on the resin blocks for the TEM, we cut $\sim 50 \mathrm{~nm}$ ultrathin sections and collected them sequentially on pioloform-coated copper slot grids to form series of 20-300 sections.

Specimens were imaged using an $80 \mathrm{kV}$ transmission electron microscope (TEM, 100CX, JEOL) at 2000-26,000×. Boutons that had been labeled with $\mathrm{DAB}$ (a uniform black substrate in the cytoplasm) were located systematically, and images were captured for analysis using a digital camera (DigitalMicrograph, Gatan). EM stacks were aligned manually in Reconstruct. For block-face imaging, pins were mounted into the 3View 2XP System (Gatan) coupled to a $1.5 \mathrm{kV}$ scanning electron microscope (GeminiSEM 300, Carl Zeiss). The surface of the pin was imaged using a backscatter detector, and then ROIs were selected based on the presence of labeled axons. ROIs were generally $20-25 \mu \mathrm{m}^{2}$ fields imaged at $6.5 \mathrm{~nm}$ resolution. A built-in microtome then cut a $50 \mathrm{~nm} \mathrm{sec-}$ tion from the surface of the pin, and the ROIs were imaged again. In this way, long series of 100-300 sections were imaged in sequence at each ROI. For series obtained using block-face imaging, we used an algorithm for alignment (GMS3.0, Gatan).

EM stacks were imported into Reconstruct, where we exhaustively measured all DAB-labeled boutons for major diameter to quantify bouton size. In cases where the entirety of the bouton was present, we also measured the bouton volume and surface area of the postsynaptic density (PSD). Some of these boutons and their postsynaptic structures were reconstructed into 3D models using the software 3D Studio Max (Autodesk) for basic smoothing. We also noted several qualitative factors in the synaptic interaction (e.g., the presence or absence of mitochondria in a bouton). We quantified the proportion of boutons that formed synapses on a $\mathrm{CBP}^{+}$postsynaptic element, as labeled by gold (appears as round, black quanta, sometimes clustered). For a comparison with features in nonlabeled pathways, we studied equivalent quantitative and qualitative characteristics in the surrounding neuropil of the imaged sites.

NR1 labeling and optical density analysis. To study the distribution of NMDARs in A25, we used an antibody for the NR1 subunit, which is present in all NMDARs. We double-labeled for NR1 and PV, CB, or CR in coronal sections of A25 (Cases BR, BW) and used CM to obtain $z$ stacks at $630 \times$ through the depth of tissue in the superficial and deep layers. Images were deconvolved, and we selected focal planes that contained $\mathrm{CBP}^{+}$-labeled neurons and pyramidal neurons for analysis.

Using ImageJ, we manually segmented each selected image, producing three binary image masks per image. The masks allowed us to extract only the pixels within cells (or neuropil regions) of interest and to exclude other aspects of the image. The first mask contained an outline of all $\mathrm{CBP}^{+}$cells, the second mask contained an outline of all pyramidal cells, and the third mask contained outlined regions of neuropil. The neuropil masks served two purposes. First, to introduce a control within each image that could be used for normalization and thus comparison across images. Second, to provide a functional benchmark with which to interpret results. The neuropil NR1 labeling is produced by a mix of intermingled neuronal elements from nearby cells that contain NMDARs. An average level of NR1 in the neuropil produces a benchmark to interpret whether the NR1 population of a particular cell type could have a pronounced effect in local signaling. Each manually segmented binary mask was then used to extract image pixels from the matched greyscale single NR1 channel image using MATLAB (RRID:SCR_001622).

To perform the optical density analysis, we normalized each extracted image so that the maximum brightness was 1 , and then the masked images were thresholded to remove the lower $25 \%$ of pixel intensities. This threshold was selected to exclude most of the nucleus of each neuron, where NR1 labeling appeared absent. Removal of the nucleus by thresholding highlighted cytoplasmic regions in each cell, where we observed the overwhelming majority of NR1 labeling. For each image, the average NR1 intensity per pixel was computed for the CBP neuron type (PV, CB, or CR), the pyramidal cell type (Pyr), and for the neuropil. We produced an average density ratio (average density within neuronal cytoplasm per cell type/average density in neuropil) for each image. For each case, this produced eight sets of density ratios: four neuronal types (PV, CB, CR, Pyr) from two laminar subdivisions, the superficial and the deep.

Data analysis and statistics. We used SPSS for data analysis and statistics (IBM, RRID:SCR_002865). We used ANOVA to determine whether there were differences among stereological estimations of the density of $\mathrm{CBP}^{+}$-labeled neurons in the laminar groups of $\mathrm{A} 25, \mathrm{~A} 32$, and A46. For analysis of A32 and neuropil bouton size (and other structural characteristics) using BM and EM measurements, we used ANOVA to determine whether there were laminar differences or differences in synaptic interactions from the axon terminals from A32 compared with structures in the surrounding neuropil. We also used ANOVA to determine whether there were differences among the $\mathrm{CBP}^{+}$ synaptic targets of A32 in A25. To determine whether PSD was correlated with bouton size, we used linear regression. For statistical analysis of the NR1 labeling, we performed a one-way ANOVA for each case, with multiple comparison tests with Bonferroni's correction.

Brightness, contrast, and saturation adjustments for photographs were conducted using ImageJ (RRID:SCR_003070; Rasband, 1997-2014) or Adobe Photoshop (RRID:SCR_014199), but no retouching was performed. Figures were prepared in Adobe Illustrator CC (RRID:SCR 010279).

\section{Results}

Figure 1 shows our experimental approach for simultaneous labeling of the serial pathways. We injected bidirectional neural tracers into A32 to retrogradely label neurons in the dlPFC and frontal pole and to anterogradely label axonal terminations in A25 (Fig. 1A). This allowed us to map the pathway from the dlPFC/frontal pole to A32 in coronal plots (Fig. 1B) and from A32 to A25 using stereological sampling (Fig. 1C). Using the same injection sites, we quantified the size and density of A32 terminations in $\mathrm{A} 25$ (Fig. $1 C$, right). We then used higher resolution approaches to study the excitatory and inhibitory postsynaptic targets of A32 terminations in A25 at the confocal and synaptic levels (Fig. 1D).

\section{Injection sites}

In 6 cases ( $n=4$ female), we injected the pregenual anterior cingulate A32 with a bidirectional neural tracer (Fig. 2A; Table 1; BDA, $n=4$, Cases AY, BG, BI, BS; LY, $n=2$, Cases BL, BN). Two injections were placed in anterior A32 (BS, AY), two in mid-level A32 (BI, BL), and two in posterior $\mathrm{A} 32(\mathrm{BL}, \mathrm{BN})$. In 1 case $(\mathrm{BG})$, the tracer spread to a small portion of medial area 9 in the fundus of the cingulate sulcus. Injection sites included all cortical layers. With the exception of case BS (Fig. 2B), all injections have been used in an unrelated previous study (García-Cabezas and Barbas, 2017).

\section{Superficial layer neurons in dlPFC and A10 project to A32, which projects to all layers of A25}

To study the serial pathways from dIPFC to A25, we characterized the first portion from the dlPFC and A10 to A32 by 

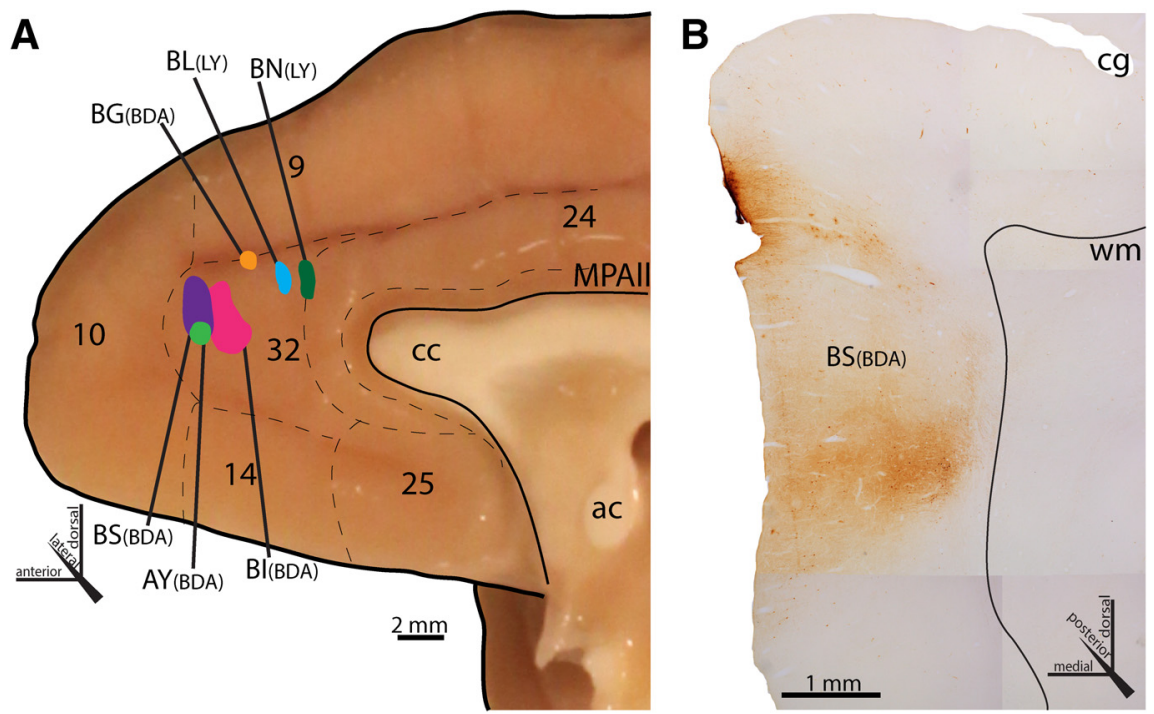

Figure 2. Injection of bidirectional tracers into A32. A, Injection sites in A32 on the medial surface of the rhesus monkey brain. Six injection sites are represented in a normalized brain space through the anterior to posterior aspects of A32. Numbers indicate cortical areas according to Barbas and Pandya (1989). B, Injection site of BDA in A32 in all layers (Case BS). Black line indicates boundary between layer VI and the superficial white matter. ac, anterior commissure; $c c$, corpus callosum; $\mathrm{cg}$, cingulate sulcus; MPAll, medial periallocortex; wm, white matter.

mapping retrogradely labeled neurons in coronal sections sampled at short equal intervals $(0.8-1 \mathrm{~mm})$. As shown in Figure $3 A$, labeled neurons in A10, A9, and A46 that project to A32 were found mostly in the superficial layers. Across cases, the proportion of neurons found in the superficial layers indicated a strong feedforward relationship from the dIPFC and A10 to A32 (Fig. 3B; A10, $78 \pm 10 \%$; A9, $74 \pm 7 \%$; A46, $80 \pm 7 \%$ ).

We then characterized the second portion of the serial pathway by mapping the terminations of A32 in A25. We used sections sampled at short equal intervals $(1 \mathrm{~mm})$ and stereologically sampled all of A25 present in each section (Fig. 3C). Using the plots from each section, we assigned indices to the density of A32 terminations in the dorsomedial, ventromedial, and orbital aspects of A25. We translated the density indices into colorcoded maps for each A32 injection site to reveal the distribution of A32 inputs in A25 (Fig. 3D).

We found that A32 terminations innervated all layers of A25 (Fig. 3E). We expressed the laminar specificity of the pathway as the ratio of the density of boutons in the deep layers to the density in all layers, finding that A32 innervated A25 in a lateral, or columnar fashion. The ratio showed that A32 terminations in A25 were roughly equivalent in the superficial and the deep layers (Fig. $3 E$; superficial, $0.962 \pm 0.05$ boutons $/ \mathrm{mm}^{3}$, deep, $1.07 \pm 0.08$ boutons $/ \mathrm{mm}^{3}$, one-tailed $t$ test, $\left.t_{(4)}=2.13, p=0.26 ; n=5\right)$.

The cortical structure of the dlPFC/A10, A32, and A25 places the serial pathway in the context of a larger Structural Model of corticocortical connections that is based on the relationship between the laminar structure of two linked cortical areas (Barbas and Rempel-Clower, 1997; for review, see Barbas, 2015; García-Cabezas et al., 2019), as summarized in Figure 3F. Figure $3 G$ depicts the cytoarchitecture of A46, A32, and A25. A46 is a eulaminate cortex with six well-defined layers, while A32 and A25 are both dysgranular cortices with impoverished layer IV. Afferent cortical connections that originate in the superficial layers (layers II-III) are classified as feedforward and are predicted when an area with better delineated laminar structure projects to an area with less delineated laminar structure. The second portion of this serial pathway, from A32 to A25, is a columnar (also known as lateral) pattern of projection, in accord with the Structural Model's prediction for two linked areas of comparable structure.

\section{Inhibitory microenvironments and $\mathrm{CB}^{+}$pyramidal neurons at the serial pathway nodes}

To place these laminar connections in the context of the unique inhibitory microenvironments of each area, we studied the density of neurons labeled for PV, CB, and CR in A25, A32, and A46. The CB antibody is known to also label some pyramidal neurons in some cortical areas (Gabbott and Bacon, 1996a; DeFelipe, 1997). Figure $4 A-C$ depicts CB neurons distributed in cortical columns of A46, A32, and A25. As shown in Figure 4D, A25 contains many $\mathrm{CB}^{+}$pyramidal neurons in the superficial layers (green arrows), although they can be distinguished from inhibitory counterparts (red arrows) using labeling intensity and morphologic features. $\mathrm{CB}^{+}$pyramidal neurons are lightly labeled, with an absence of labeling in the nucleus, and are pyramidal in shape. On the other hand, $\mathrm{CB}^{+}$inhibitory neurons appear uniformly dark and have a nonpyramidal shape and often appear multipolar (Fig. 4E). Staining for GAD-67 (a synthetic enzyme for GABA; Fig. $4 F$ ) and double-labeling for GABA and CB (Fig. $4 G-I$ ) support the classification of $\mathrm{CB}$ neurons based on labeling intensity and morphologic criteria. This approach made it possible to disambiguate the presumed excitatory and inhibitory $\mathrm{CB}^{+}$ neurons at the serial pathway nodes. Stereological sampling and estimation revealed that the density of $\mathrm{CB}^{+}$presumed pyramidal neurons was higher in superficial than in deep layers (Fig. 4J,K) but highest in A25 among the three cortical areas (Fig. $4 L$; all layers, A25: $9711 \pm 4026$ neurons $/ \mathrm{mm}^{3}, \mathrm{~A} 32: 4851 \pm 1802$ neurons $/ \mathrm{mm}^{3}, \mathrm{~A} 46: 1227 \pm 680$ neurons $/ \mathrm{mm}^{3}$; one-way ANOVA, $\left.F_{(2,6)}=2.730, p=0.144\right)$, which is in accord with previous findings (Dombrowski et al., 2001).

The distribution of $\mathrm{PV}$, presumed inhibitory $\mathrm{CB}$, and $\mathrm{CR}$ neurons in the layers of A25, A32, and A46 is shown in Figure 5A-D. The density of PV neurons across laminar groups in A25, A32, and A46 has been previously published (Garcia-Cabezas et al., 2017), and is included here for completeness. We found that PV neuron density in the middle to deep layers of A46 was almost double that of $\mathrm{A} 32$ and $\mathrm{A} 25$, while $\mathrm{CB}$ and $\mathrm{CR}$ neuron densities were comparable across areas (Fig. 5E; A25: $3736 \pm 575$ neurons $/ \mathrm{mm}^{3}$; A32: $3522 \pm 468$ neurons $/ \mathrm{mm}^{3}$; A $46: 7613 \pm 377$ neurons $/ \mathrm{mm}^{3}$; one-way ANOVA, $\left.F_{(2,6)}=22.997, p=0.002\right)$. PV neurons are localized mostly in the middle to deep layers in all areas, while CR and darkly stained, presumed inhibitory, CB neurons are localized more preferentially in the superficial layers. 
A

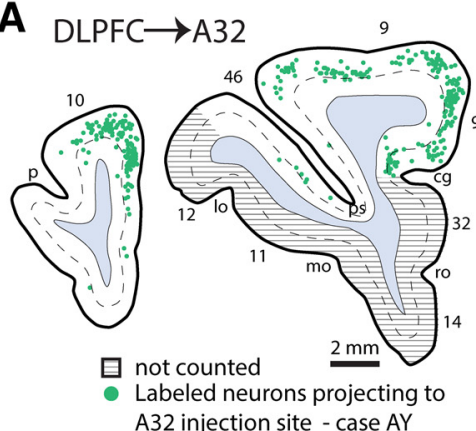

B

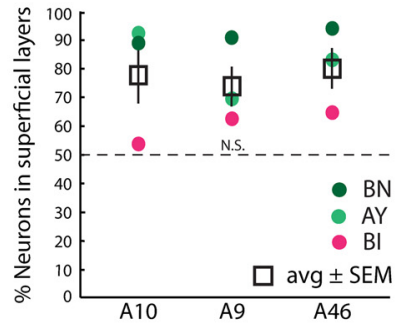

C

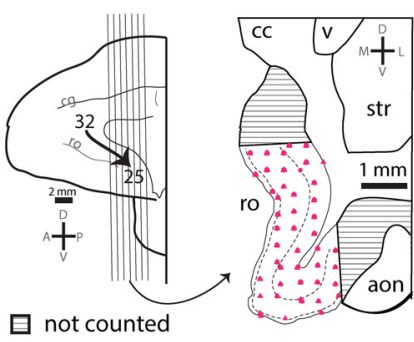

- Labeled boutons from A32 - case BI
D

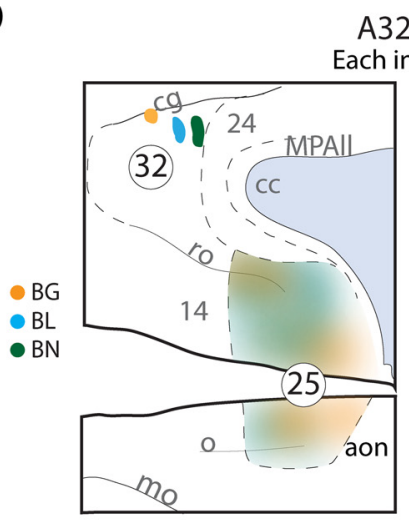

A32 $\rightarrow$ A25

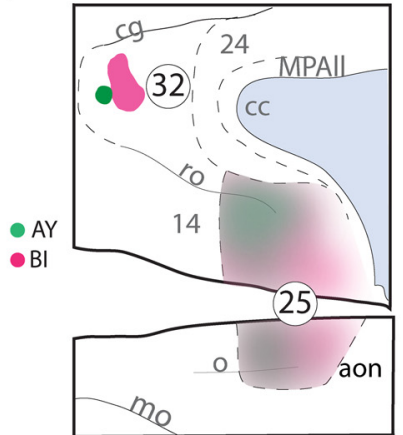

E

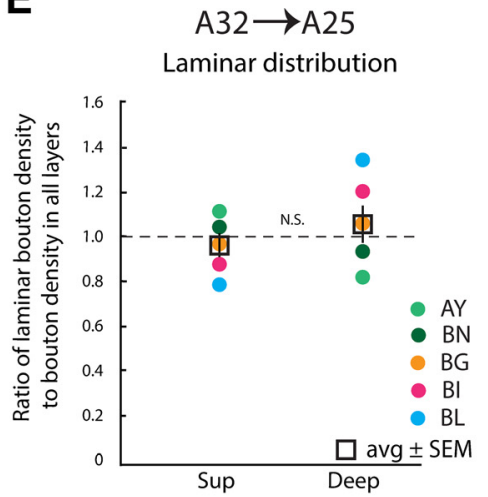

F $\quad \mathrm{DLPFC} \rightarrow \mathrm{A} 32 \rightarrow \mathrm{A} 25$

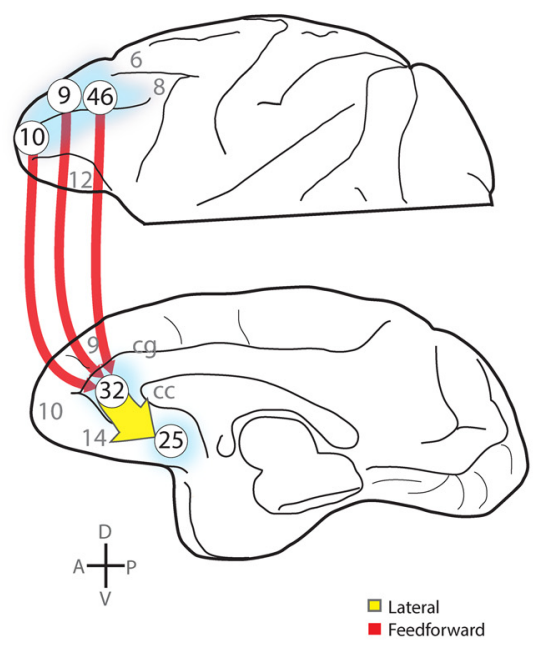

G

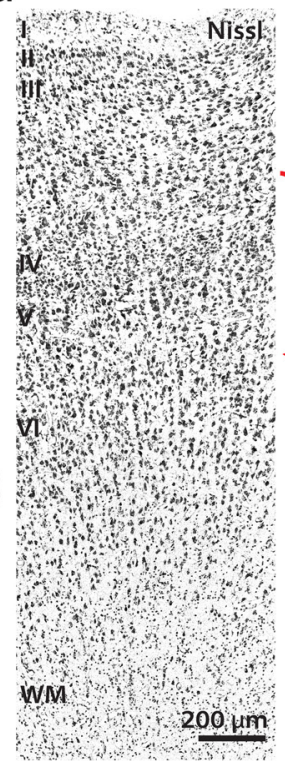

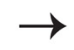

$\rightarrow$

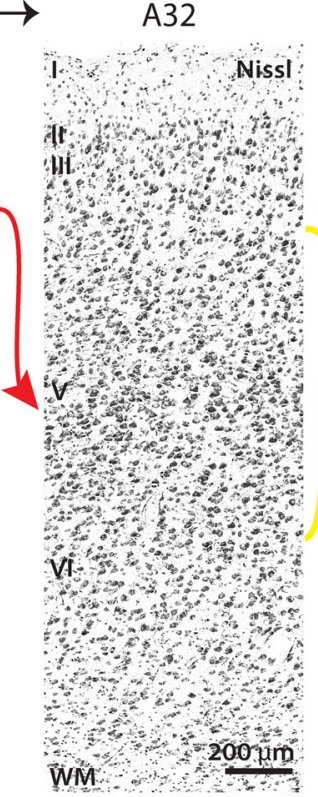

$\rightarrow$

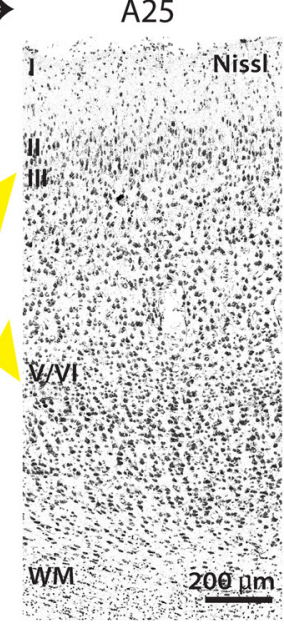

Figure 3. System-level mapping of serial pathway from dIPFC and A10 to A25 through A32. A, Coronal sections show plots of neurons (green) projecting to A32 injection site. Projection neurons are distributed mostly in the superficial layers. Areas marked by hatching were not mapped in this study. $\boldsymbol{B}$, Distribution of retrogradely labeled neurons directed to A32 injection site from areas in IIPFC and A10, expressed as percent of neurons found in the superficial layers. C, Sampling of coronal sections for stereological computation of A32 termination density in A25. Sections were sampled at $1 \mathrm{~mm}$ through the extent of A25. Laminar categories of A25 were outlined, and stereological sampling was conducted in small fields of $25 \mu \mathrm{m}^{2}$. Markers used to count A32 terminations were enlarged so the fields are visible. D, Distribution of A32 terminations in subregions of A25, shown in color by each injection site. Stronger color represents denser distribution of A32 axonal terminations. $\boldsymbol{E}$, Laminar distribution of A32 terminations in A25, expressed as the ratio of bouton density in a laminar category to the bouton density in all layers. $\boldsymbol{F}$, Color-coded summary schematic of laminar pattern of connections in the serial pathway. Connections between cortical areas are in accordance with the Structural Model (Barbas and Rempel-Clower, 1997). Connections from the six-layered dIPFC/A10 flow to A32 in a feedforward pattern (red), from the superficial layers of dIPFC/A10 to the deep layers of A32. A32 and A25 both contain fewer than six layers, and have a lateral pattern of connections (yellow), meaning that the superficial and deep layers of both areas engage in connections between them. G, Photomicrographs of coronal sections in A46, A32, and A25 stained for Nissl. A46 has six distinct layers, while A32 and A25 do not. Color-coded arrows indicate the laminar connections in the serial pathway, and follow the color key represented in $\boldsymbol{F}$. Roman numerals indicate cortical layers. Numbers indicate cortical areas according to Barbas and Pandya (1989). aon, anterior olfactory nucleus; cc, corpus callosum; cg, cingulate sulcus; lo, lateral orbital sulcus; mo, medial orbital sulcus; p, principal sulcus; rh, rhinal sulcus; ro, rostral sulcus; str, striatum; sup, superficial; $\mathrm{v}$, ventricle; WM, white matter. 

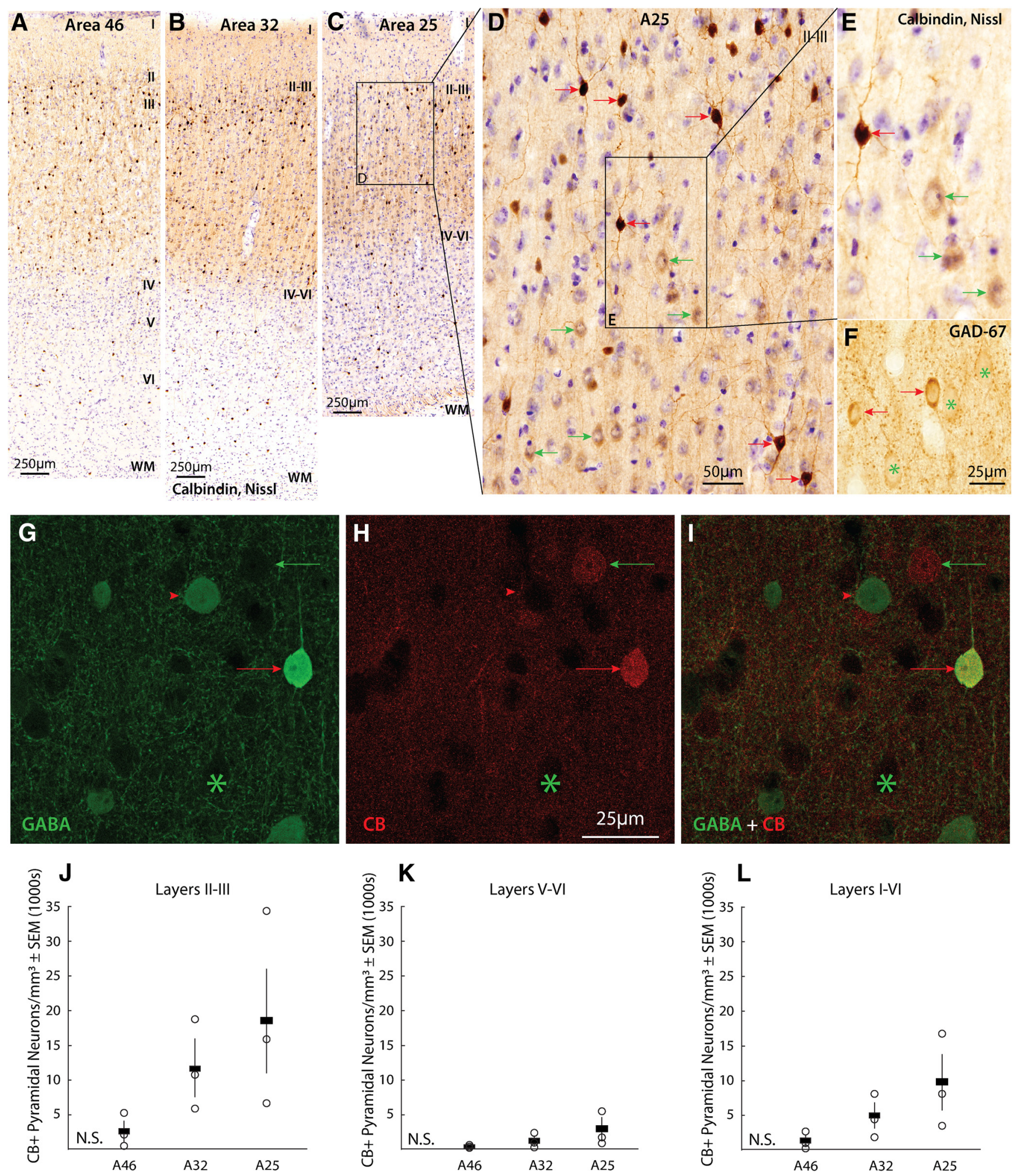

Figure 4. CB-labeled neurons in A25. A, Photomicrograph of coronal section in A46 immunolabeled for CB (brown) and counterstained with Nissl. $\boldsymbol{B}$, Photomicrograph of coronal section in A32 immunolabeled for CB and counterstained with Nissl. C, Photomicrograph of coronal section in A25 immunolabeled for CB and counterstained with Nissl. D, Inset, Pyramidal-shaped (excitatory) neurons lightly labeled with CB (green arrows) and multipolar-shaped presumed inhibitory neurons that have a uniform dark (B label (red arrows) in the superficial layers of $A 25$. $\boldsymbol{E}$, Higher-magnification inset, Pyramidal-shaped neurons lightly labeled with CB (green arrows) and multipolar-appearing neuron with a uniform dark (B label (red arrow) in the superficial layers of A25. F, Photomicrograph of the superficial layers of A25 immunolabeled with GAD-67, a marker of inhibitory neurons. Red arrows indicate neurons with cytoplasm strongly labeled by GAD-67. Green asterisks indicate morphologically distinct pyramidal shaped (excitatory) neurons that show GAD-67 puncta targeting their cell bodies but an otherwise absence of GAD-67 labeling. G, The superficial layers of A25 immunolabeled for GABA show inhibitory neurons labeled by green fluorophores (red arrow, red arrowheads). Empty spaces represent the cell bodies of non-GABA-labeled neurons (green arrow, green asterisk). $\boldsymbol{H}$, The same field of view (FOV) in the superficial layers of A25 immunolabeled with CB shows a darkly labeled CB neuron (red arrow), a lightly labeled neuron above it (green arrow), and empty spaces representing cell bodies of non-CB-labeled neurons (red arrowhead, green asterisk). $I$, Merged GABA and (B. Red arrow indicates an inhibitory neuron labeled for both GABA and CB. Red arrowhead indicates a GABA-positive neuron that is CB-negative, presumably a PV or CR inhibitory neuron. Green arrow indicates 
Overall, these findings depict a changing laminar population of inhibitory neurons at each node, and demonstrate that limbic prefrontal areas A32 and A25 are comparatively impoverished in middle-layer PV inhibitory neuron density as compared with A46 (Fig. 5E). Density data for PV, CB, and CR neurons is comparable to stereological estimates from another study (Gabbott and Bacon, 1996b).

\section{The inhibitory targets of A32 in A25: CM}

After mapping the serial pathway and characterizing the inhibitory neurons found in all nodes, we used higher resolution methods to focus on the second portion of the serial pathway. First, we examined the relationship between large populations of A32 terminations and their appositions to inhibitory processes in A25 by obtaining 3D stacks at the sampling sites in Figure $6 \mathrm{~A}$. An apposition is a point of near contact between an axonal termination and $\mathrm{CBP}^{+}$structure (Fig. $6 B$ ).

Analysis of 3D stacks obtained by CM revealed that $\sim 25 \%$ of A32 terminations were apposed to CBP labeled structures and thus presumed to be on inhibitory neurons. Among these, A32 boutons preferentially targeted $\mathrm{CR}$ neuronal processes in all layers, and were more likely to target PV neuronal processes in the deep layers than in the superficial layers.

The breakdown of A32 targets is shown in Figure 6C. In the superficial layers, A32 targeted very few PV neuronal processes $(2 \pm 0 \%)$, a moderate number of $\mathrm{CB}$ processes $(8 \pm 2 \%)$, and more $\mathrm{CR}$ processes $(14 \pm 2 \%)$ than $\mathrm{PV}$ and $\mathrm{CB}$ combined $(n=$ 6535 boutons, Cases BI, BS; one-way ANOVA, $F_{(2,3)}=15.216$, $p=0.027$; post hoc Tukey's test, $\mathrm{PV}$ vs CR, $p=0.024)$. The remaining $76 \%$ of $\mathrm{A} 32$ terminations were presumed to appose unlabeled excitatory neuronal processes because together PV, $\mathrm{CB}$, and CR label almost the entirety of cortical inhibitory neurons (for review, see Barbas et al., 2018).

In the middle and deep layers of A25, A32 terminations targeted a moderate number of PV $(6 \pm 2 \%)$ and $\mathrm{CB}(6 \pm 2 \%)$ neuronal processes, but preferentially targeted CR neuronal processes $(13 \pm 1 \%)$ at double the rate $(n=5840$ boutons, Cases BI, BS; one-way ANOVA, $\left.F_{(2,3)}=5.444, p=0.100\right)$. The remaining $74 \%$ of A32 terminations were presumed to innervate excitatory neurons in A25. These findings reflect the higher prevalence of PV neurons in the middle to deep layers and CB neurons in the superficial layers (Fig. 5). There were no significant differences in the aggregate number of inhibitory versus excitatory elements targeted by A32 terminations in the superficial versus deep layers (one-way ANOVA, $F_{(1,2)}=0.163, p=0.725$ ).

In summary, A32 terminations were most likely to appose excitatory targets. Among targets on inhibitory neurons, more were on CR than PV or CB elements in all layers (Fig. $6 C$ ). In the middle and deep layers, A32 terminations were more likely to appose PV neurons than in superficial layers (one-tailed $t$ test, $t_{(3)}=5.28$,

\footnotetext{
a lightly labeled CB neuron that is GABA-negative, presumably a CB-positive pyramidal neuron. Green asterisk indicates the shadow of a cell body that is both GABA- and CB-negative, presumably a CB-negative excitatory neuron. Roman numerals indicate cortical layers. $J$, Average neuronal density of $\mathrm{CB}^{+}$presumed excitatory neurons in superficial layers of $A 46$, $A 32$, and $A 25$ based on computations performed from stereological sampling. Circles represent values from individual cases. Data are mean $\pm \mathrm{SE}$. $\boldsymbol{K}$, Average neuronal density of $\mathrm{CB}^{+}$ presumed excitatory neurons in deep layers of $A 46, A 32$, and $A 25$ based on computations performed from stereological sampling. Circles represent values from individual cases. Data are mean \pm SE. $\boldsymbol{L}$, Average neuronal density of $\mathrm{CB}^{+}$presumed excitatory neurons in all layers of $A 46, A 32$, and $A 25$ based on computations performed from stereological sampling. Circles represent values from individual cases. Data are mean \pm SE. WM, White matter.
}

$p=0.007)$. The A32 pathway apposed about an equal number of CR postsynaptic sites in the upper and deep layers (Fig. $6 \mathrm{C}$, green).

\section{The synaptic targets of A32 in A25: EM}

We then used the highest resolution approach to examine synaptic interactions of A32 in A25 in long series of ultrathin sections cut through A25 at the sampling sites shown in Figure 7A. Using classical criteria to identify synapses, boutons, and postsynaptic structures (Peters et al., 1991), we analyzed the postsynaptic targets of A32 terminations in the superficial and deep layers of A25. The majority of A32 terminations targeted unlabeled spines on densely spiny dendrites $(\sim 80 \%)$, which are characteristic of excitatory neurons. A smaller, but significant, proportion of A32 boutons terminated on $\mathrm{CBP}^{+}$structures, most commonly on their dendritic shafts, as cortical inhibitory neurons are smooth, or sparsely spiny (Fig. 7B) (DeFelipe, 1997). Of the $\mathrm{CBP}^{+}$postsynaptic targets, A32 terminations mainly targeted CR neurons in the superficial layers and PV neurons in the deep layers.

In the superficial layers, A32 terminations formed synapses on $\mathrm{CR}$ sparsely spiny dendrites $(10 \pm 0.5 \%)$, followed by $\mathrm{CB}$ $(7 \pm 0.4 \%)$ and PV $(4 \pm 0.4 \%)$ sparsely spiny dendrites (Fig. $7 C$, one-way ANOVA, $F_{(2,5)}=56.582, p=0.004$; post hoc Tukey's test, $\mathrm{PV}$ vs $\mathrm{CB} p=0.023$, $\mathrm{PV}$ vs $\mathrm{CR} p=0.004, \mathrm{CB}$ vs $\mathrm{CR} p=0.03$ ). These results largely matched the trend from appositional analysis in superficial layers, which also showed a preference of A32 for $\mathrm{CR}$ in the superficial layers. In 1 case (BI), we observed that two of the A32 terminations formed synapses on $\mathrm{CB}^{+}$soma in the superficial layers (Fig. $7 C$, right).

In the deep layers, A32 terminations formed synapses on aspiny or sparsely spiny dendrites labeled for PV (10 $\pm 0.2 \%)$, followed by $\mathrm{CR}(5 \pm 0.8 \%)$ and $\mathrm{CB}(4 \pm 0.8 \%)$, as shown in Figure $7 D$ (one-way ANOVA, $F_{(2,5)}=20.03, p=0.018$; post hoc Tukey's test, PV vs CB $p=0.022$, PV vs CR $p=0.028, \mathrm{CB}$ vs CR $p=0.89)$. These figures reflect more PV synapses and fewer CR synapses than the respective appositions described above, and may be because of the differences in the resolution of the methodologies used. In 1 case (BI), we observed that one of the A32 terminations formed a synapse on a $\mathrm{CR}^{+}$soma in the deep layers (Fig. $7 D$, right, dark green).

Figure 8 shows a 3D-rendered reconstruction of all A32 axonal terminations (blue) and their postsynaptic targets in an ROI in the deep layers of A25 (200 serial $35 \mu \mathrm{m} \times 35 \mu \mathrm{m}$ sections, each $50 \mathrm{~nm}$ thick, Case BI). We depict this site for complete reconstruction to illustrate the large number of synaptic interactions in this pathway. Within this small termination site, A32 formed a synapse (Fig. $8 A$, A32 termination, blue) on a CR soma (Fig. $8 A$, pink), an unlabeled aspiny dendrite (Fig. $8 B$, red, likely a PV dendrite), and another 20 synapses on spiny dendrites, presumed to be excitatory (Fig. 8C, light green). The number of synapses evident even at very high resolution suggests that A32 has a major impact on A25. The only other system in which we have seen such a high density of labeled synapses at high resolution was in the pathway from the amygdala to the magnocellular mediodorsal thalamic nucleus (Timbie et al., 2020), although the overall patterns were distinct in these diverse pathways.

\section{Comparison of A32 axonal terminations with unlabeled synapses in the surrounding neuropil}

Synaptic efficacy is governed by several factors. One important factor is the size of an axonal termination, and thus its input weight into the local circuitry. Bouton size is related to the probability of multivesicular release on stimulation (Stevens, 2004; Germuska et al., 2006; for review, see Rollenhagen and Lubke, 2006). 
A
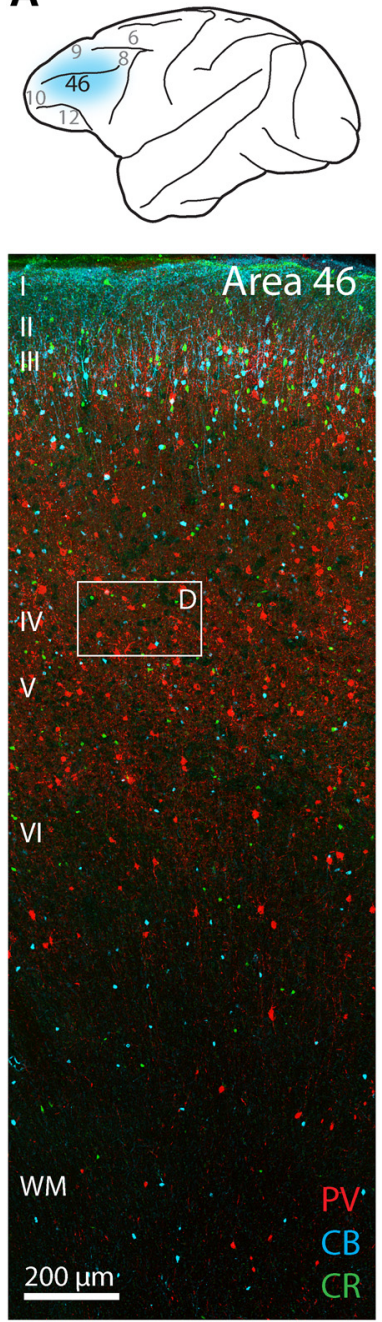

F

$F$

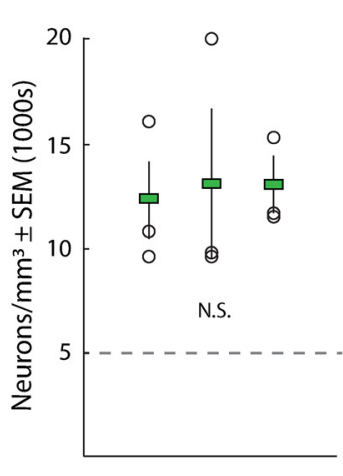

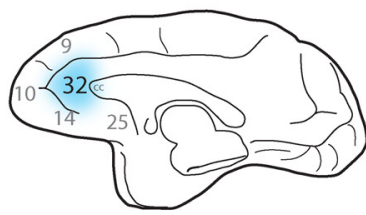
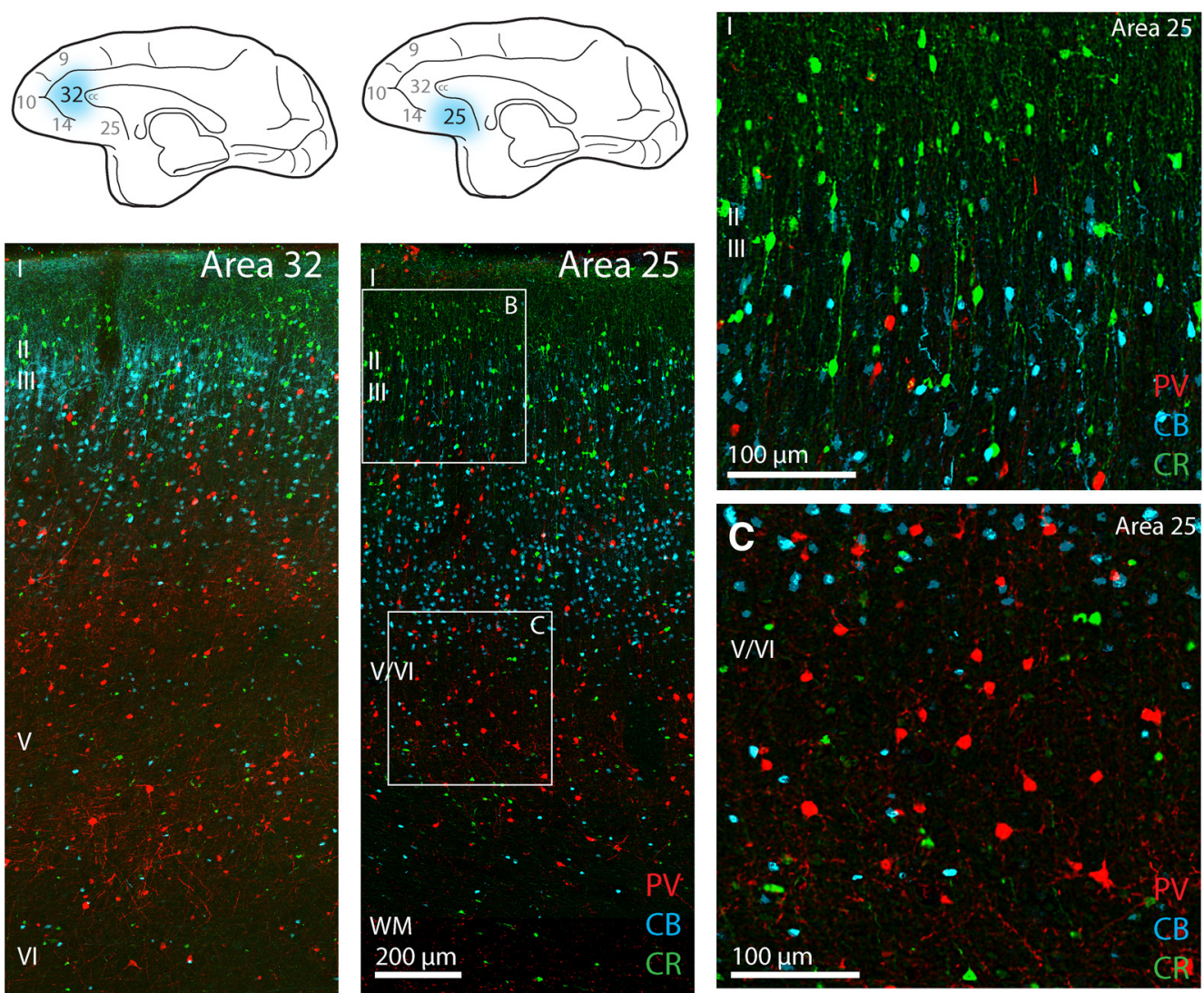

$200 \mu \mathrm{m}$

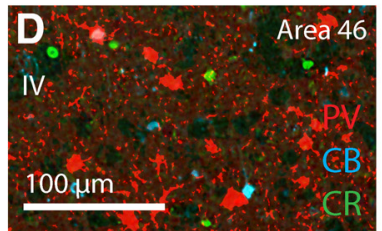

$200 \mu \ddot{m}$

G

Layers II-III

H Layer IV

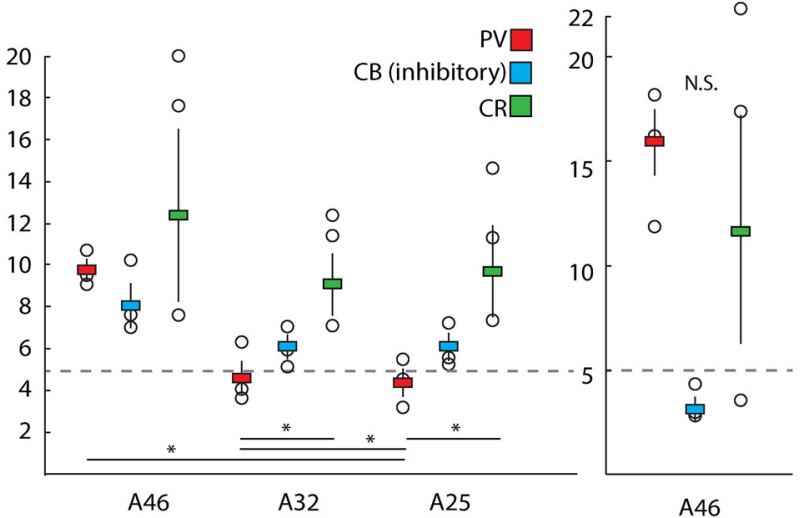

E

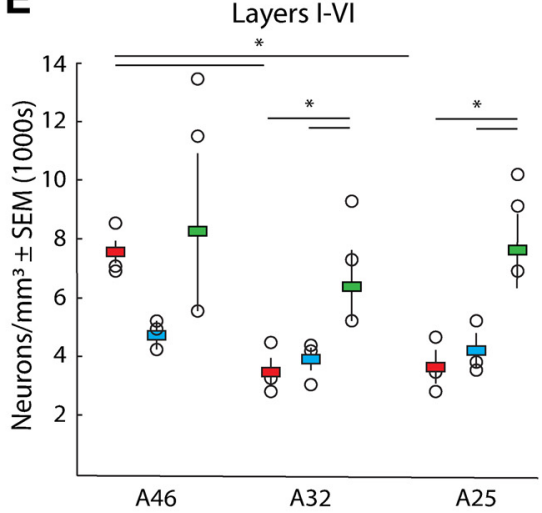

Layers V-VI

Figure 5. The distribution of $P V, C B$, and $C R$ neurons in $A 46, A 32$, and $A 25$. $A$, Cortical columns photographed from coronal sections of $A 46$, $A 32$, and $A 25$ after immunofluorescence labeling for PV (red), CB (cyan), and CR (green). Note the increase in CB labeling in the superficial layers from A46 to A25, which is indicative of the increase in CB pyramidal neuron labeling. $B$, Higher-magnification inset of the superficial layers of A25 shows a dominance of $C B$ and $C R$ neurons. Layer I contains only CR neurons. $C$, Higher-magnification inset of the mid-to deep layers of A25 shows the preponderance of PV neurons. $\boldsymbol{D}$, Higher-magnification inset of the middle layers of A46. Note the dominance of PV neurons. $\boldsymbol{E}$, Quantification of PV, CB, and CR neuron density in layers I-VI of each area using unbiased stereological sampling. $\boldsymbol{F}$, Quantification of CR neuron density in layer I of each area using unbiased stereological sampling. $\mathbf{G}$, Quantification of PV, CB, and CR density in layers II-III of each area using unbiased stereological sampling. $\boldsymbol{H}$, Quantification of PV, CB, and CR density in layer IV of A46 using unbiased stereological sampling. $I$, Quantification of PV, CB, and CR density in layers V-VI of each area using unbiased stereological sampling. Roman numerals indicate cortical layers. Colored rectangles represent averages. Black circles represent individual cases. ${ }^{*} p<0.05$. WM, White matter. 
A

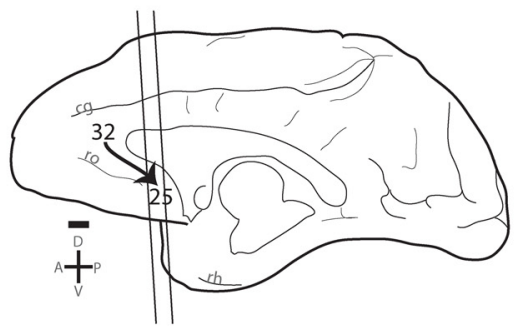

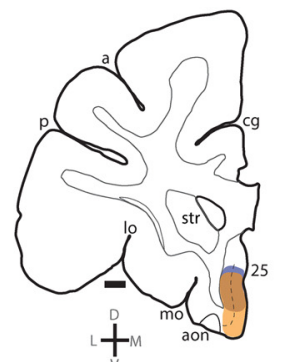

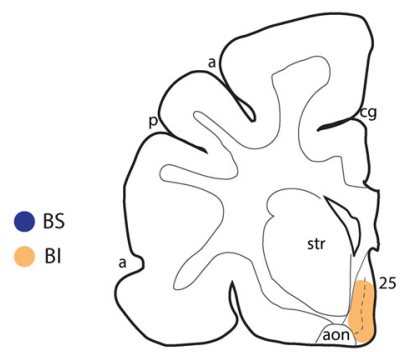

B
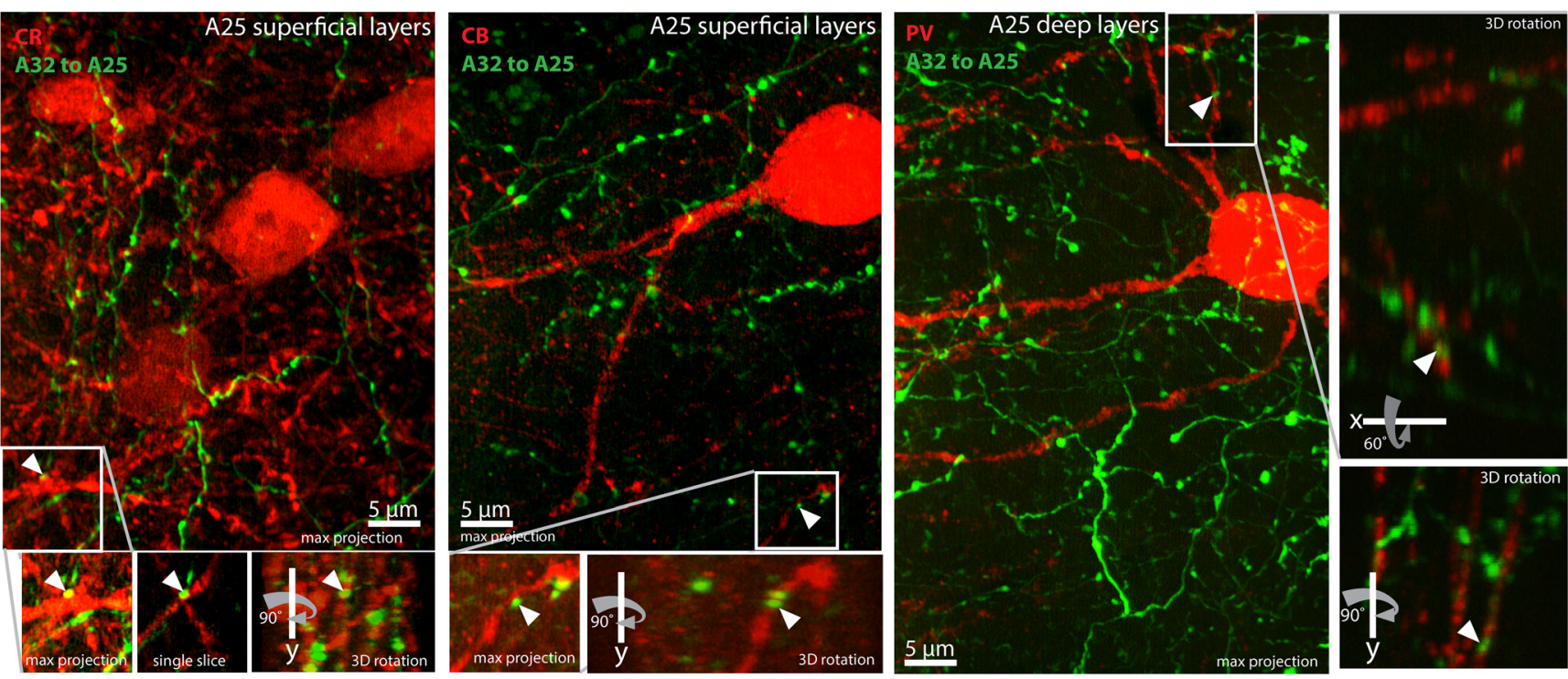

\section{Appositions to Inhibitory Processes}

\section{D}

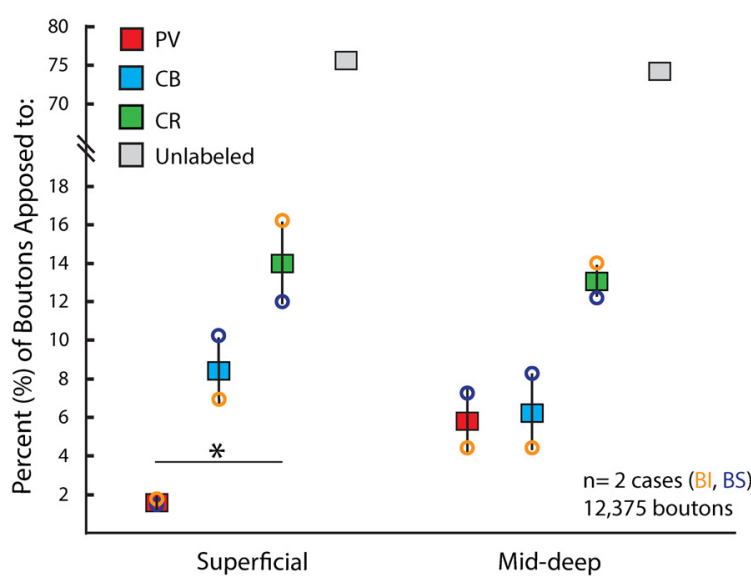

A32

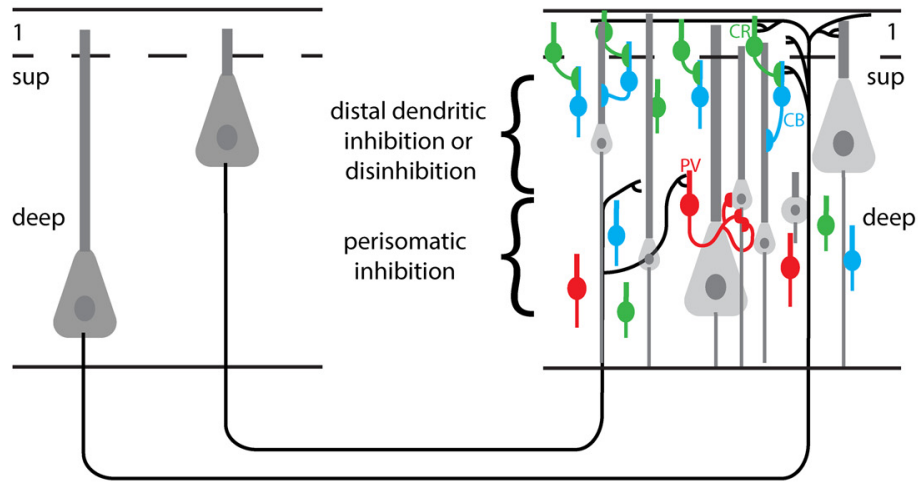

Figure 6. Appositions between $\mathrm{A} 32$ axons and $\mathrm{CBP}^{+}$structures in $\mathrm{A} 25$ as obtained via $\mathrm{CM}$. $\mathrm{A}$, Normalized brain space depicting the regions sampled for appositional analysis (Cases $\mathrm{BI}$, $\mathrm{BS}$ ). Left, The medial surface of the rhesus monkey brain. Right, Vertical lines indicate the sampling plane for the coronal sections sampled. Temporal pole not shown in coronal sections. Scale bar, $2 \mathrm{~mm}$. B, Photomicrographs of $z$ stack maximum projections taken through coronal sections of A25 laminar regions represent A32 terminations (green) and (BPP ${ }^{+}$-labeled neurons (red). A maximum projection is all the images in a $z$ stack collapsed together. Left, $\mathrm{A} 32$ terminations interact with $\mathrm{CR}^{+}$neuronal processes in the superficial layers. Insets, The selection adjusted for brightness and contrast, a single focal plane depicting an apposition between the $\mathrm{A} 32$ bouton and a $\mathrm{CR}^{+}$dendrite, and a rotated $3 \mathrm{D}$ rendering of the selection depicting that the presynaptic and postsynaptic sites are apposed in all dimensions. Middle, $\mathrm{A} 32$ terminations interact with $\mathrm{CB}^{+}$neuronal processes in the superficial layers. Insets, A higher magnification of the selection adjusted for brightness and contrast and a 3D-rendered rotation to show that the $\mathrm{A} 32$ termination apposes the $\mathrm{CB}^{+}$dendrite. Right, $\mathrm{A32}$ terminations interact with $\mathrm{PV}^{+}$neuronal processes in the deep layers. Insets, 3D-rendered rotations to show that the $\mathrm{A} 32$ termination apposes the $\mathrm{PV}^{+}$dendrite. C, Average proportion (colored boxes) of A32 boutons apposing $\mathrm{CBP}^{+}$neuronal processes in the superficial and deep layers across 2 cases (BI and BS). Colored circles represent individual cases. Data are mean $\pm S D$. ${ }^{*} p=0.05$. D, Summary schematic of the inhibitory microcircuitry in the cortical column of A25. a, arcuate sulcus; aon, anterior olfactory nucleus; cg, cingulate sulcus; lo, lateral orbital sulcus; mo, medial orbital sulcus; p, principal sulcus; rh, rhinal sulcus; ro, rostral sulcus; str, striatum. 
A

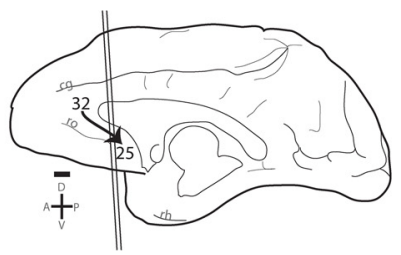

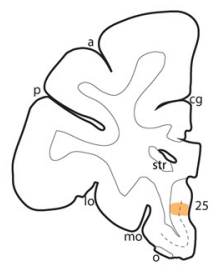

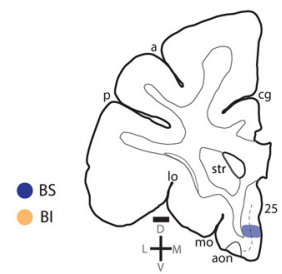

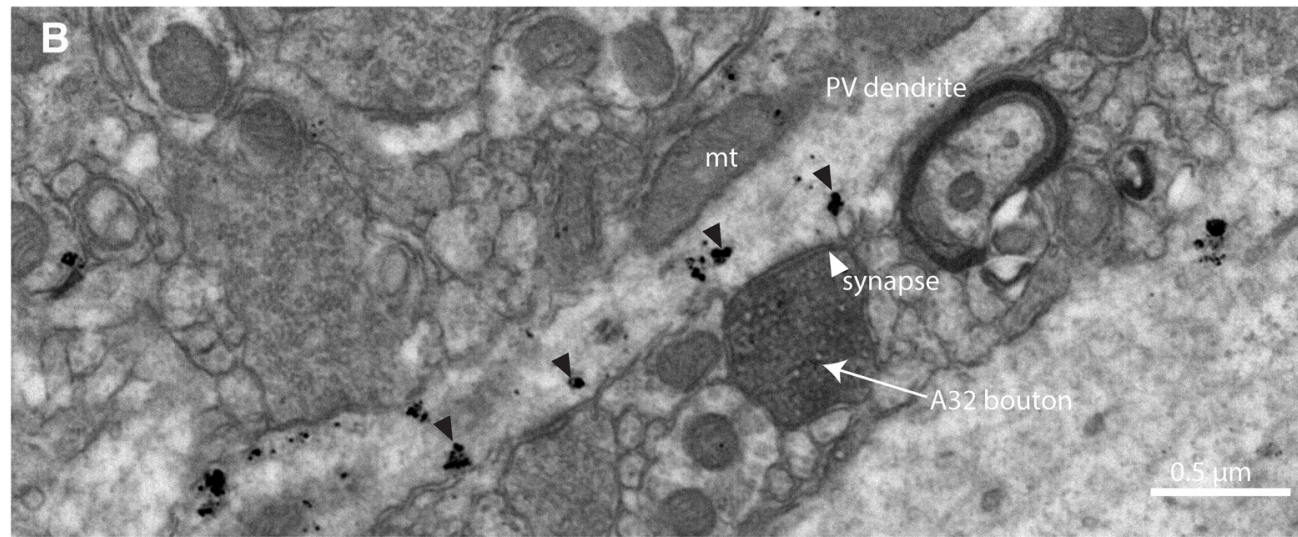

C Synapses on sparsely spiny dendrites

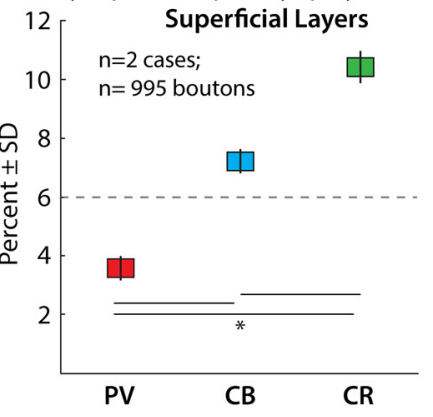

Postsynaptic Targets in Superficial Layers
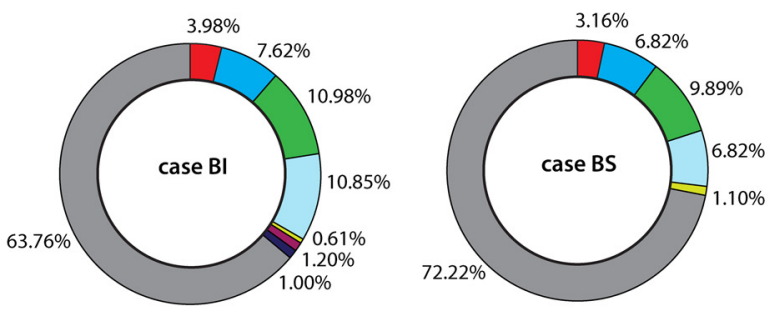

$\square$ PV sparsely spiny dendrite $\square$ PV spines, indeterminate dendrite $\square$ CB soma $\square$ CB sparsely spiny dendrite $\square$ CB spines, spiny dendrite
$\square$ CR sparsely spiny dendrite $\square$ CR spines, indeterminate dendrite $\quad$ CR soma
Unlabeled

D Synapses on sparsely spiny dendrites

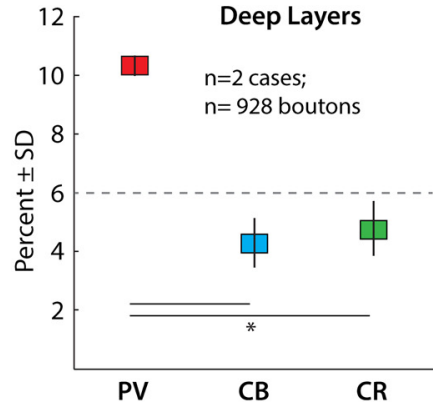

Postsynaptic Targets in Deep Layers
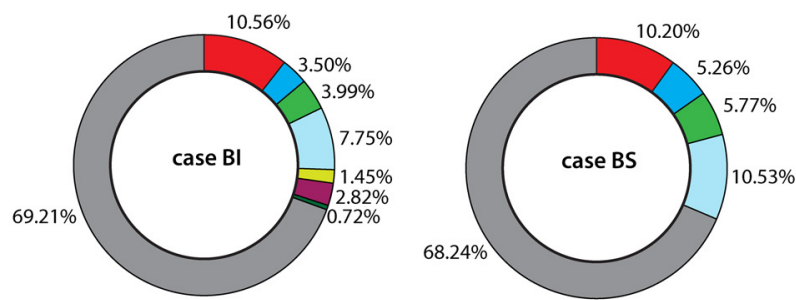

Figure 7. Postsynaptic targets of A32 terminations in A25 obtained using synaptic analysis via EM. A, Normalized brain space depicting the regions sampled for synaptic analysis (Cases BI, BS). Left, The medial surface of the rhesus monkey brain. Right, Vertical lines indicate the sampling plane for the coronal sections sampled. Scale bar, 2 mm. $\boldsymbol{B}$, Photomicrograph of an A32 axon terminal labeled with DAB (uniform dark precipitate, white arrow) that forms a synapse (white arrowhead) on a $\mathrm{CBP}^{+}$dendritic shaft in $\mathrm{A} 25$ that is positive for PV, which was labeled with gold (small black quanta, sometimes clustered, black arrowheads). C, Pattern of A32 postsynaptic targets in the superficial layers of A25. Left, Average across 2 cases (BI, BS). Right, Breakdown in individual cases. If an $\mathrm{A} 32$ bouton formed a synapse on the shaft or spine of a $\mathrm{CBP}^{+}$sparsely spiny dendrite, these interactions were presumed to be with an inhibitory postsynaptic structure. If spines could not be traced back to their dendrite for classification as sparsely spiny or densely spiny, and thus presumed inhibitory or excitatory respectively, we grouped them in a separate "indeterminate" category, which was very low for both PV and CR. Synaptic interactions between $A 32$ and $\mathrm{CBP}^{+}$spines on densely spiny dendrites, which were common for $C B$ but very rare for PV or $C R$, were grouped in their own category. D, Pattern of A32 postsynaptic targets in the deep layers of A25. Left, Average across 2 cases (BI, BS). Right, Breakdown in individual cases. ${ }^{*} p<0.05$. a, arcuate sulcus; aon, anterior olfactory nucleus; $\mathrm{cg}$, cingulate sulcus; lo, lateral orbital sulcus; mo, medial orbital sulcus; mt, mitochondria; 0 , olfactory tubercule; p, principal sulcus; rh, rhinal sulcus; ro, rostral sulcus; str, striatum.

To study the dimensions of A32 terminations in A25, we combined measurements obtained from two imaging modalities and scales (Fig. 9; BM and EM). Using the sections prepared for BM to map the second portion of the serial pathway from A32 to A25, we measured the major diameter of a large sample size of
A32 axonal terminations in systematically sampled fields of view at different focal planes $(1000 \times, z$ step of $0.5 \mu \mathrm{m})$ in the superficial and deep layers of A25. Then, we used the ultra-thin $(50 \mathrm{~nm})$ series prepared for EM analysis to measure both the major diameter and volume of A32 terminations in A25. We 


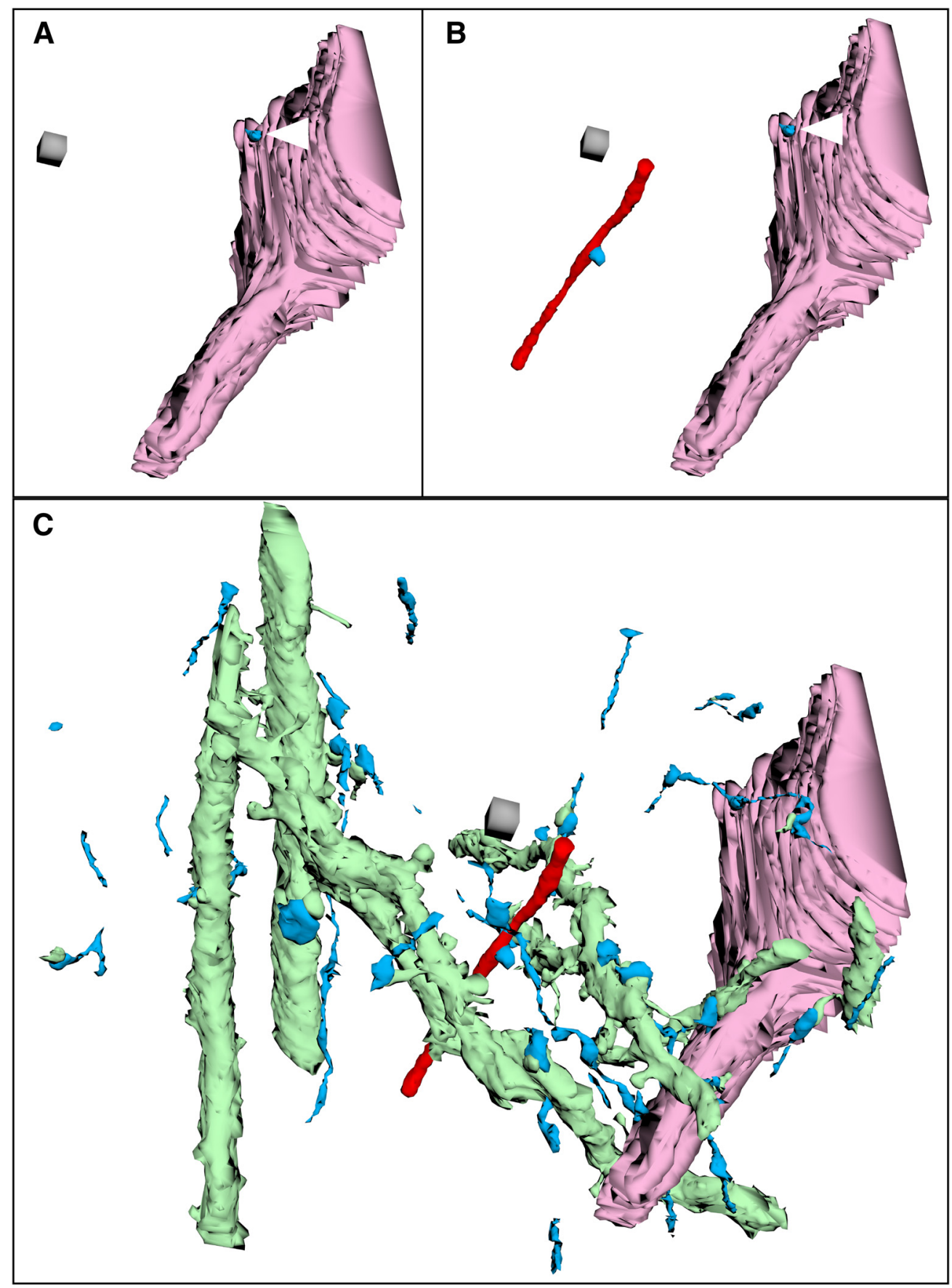

Figure 8. 3D reconstruction of an A32 terminal field in A25 from serial EM. 3D reconstruction of an ROl (200 serial sections spanning $10 \mu \mathrm{m}$ ) in the deep layers of A25 that contained 22 individual A32 terminations, demonstrating the extraordinary density of this pathway, even using the highest resolution methods. This ROl was double-labeled using immunohistochemistry for EM. A32 terminations were labeled with DAB, and CR was labeled with gold. $\boldsymbol{A}$, An A32 axon terminal (blue, white triangle) forming a synapse on a $\mathrm{CR}^{+}$soma (pink). $\boldsymbol{B}$, The same F0V but with the addition of another $\mathrm{A} 32$ axon terminal (blue) forming a synapse on an inhibitory dendrite (red). This dendrite was not $\mathrm{CR}^{+}$, and was likely a PV ${ }^{+}$dendrite. The dendrite is aspiny, or smooth, which is typical of the dendrites of inhibitory neurons. C, The full ROl containing all reconstructed structures. All axons from A32, including their terminal boutons, are labeled in blue. All postsynaptic structures receiving synapses from A32 terminations were reconstructed to the extent possible. Light green structures are spiny, presumed excitatory neuronal elements in A25. Green and blue represent excitatory neuronal processes. Red or pink represents inhibitory neuronal processes. Scale box, $1 \mu \mathrm{m}^{3}$.

also used the long series to sample the presumed excitatory boutons in the surrounding A25 neuropil (e.g., Fig. 9A, blue shading) to compare with A32 boutons (e.g., Fig. $9 A$, orangebrown shading) in A25. This analysis provides an indication of the synaptic efficacy of A32 axonal terminations compared with other excitatory pathways forming synapses in the local circuitry.

Figure $9 B$ shows the results from the combined analysis of the major diameter of A32 axonal terminations. Results from these two independent methods showed comparable A32 bouton size in superficial and deep layers (BM, deep: $0.99 \pm 0.03 \mu \mathrm{m} ; \mathrm{BM}$, sup: $0.98 \pm 0.04 \mu \mathrm{m}$; EM, sup: $1.04 \pm 0.03 \mu \mathrm{m}$; deep, EM, deep: $1.08 \pm 0.12 \mu \mathrm{m}$; two-way ANOVA, $\left.F_{(3,6)}=0.673, p=0.60\right)$. Mean diameters of $\mathrm{A} 32$ boutons fell in the normal range for corticocortical connections in other pathways (Medalla and Barbas, 2010; Bunce et al., 2013; García-Cabezas and Barbas, 2017).

The next analysis addressed the question of whether the A32 pathway was similar or different from other unlabeled 


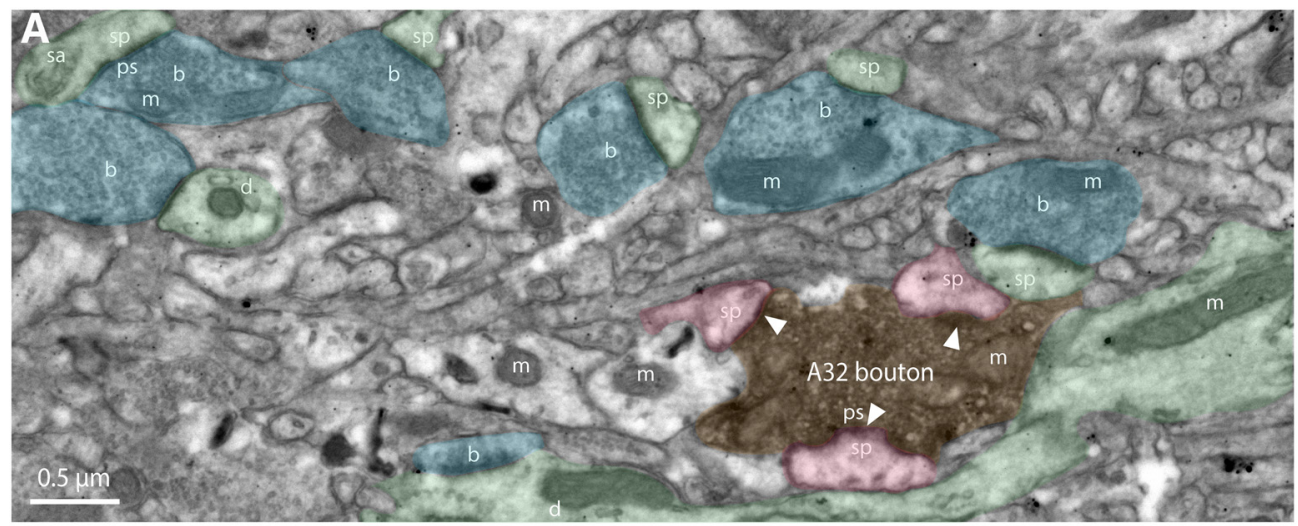

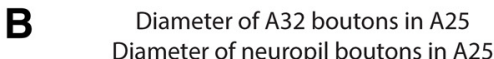

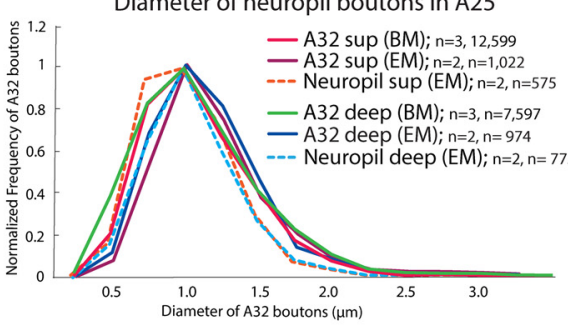

E

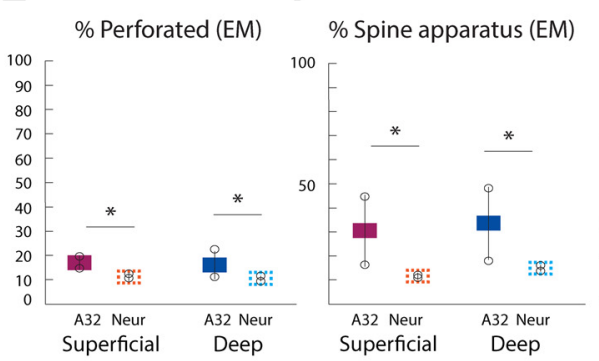

I

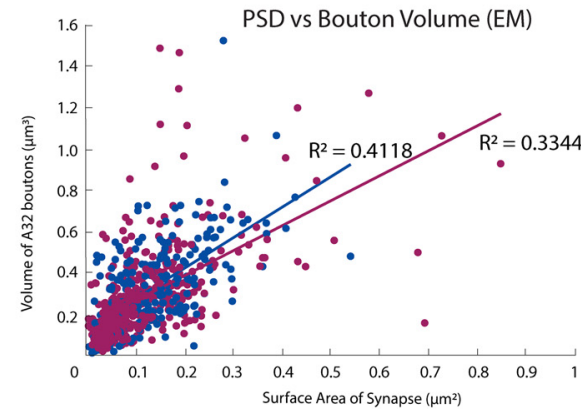

C

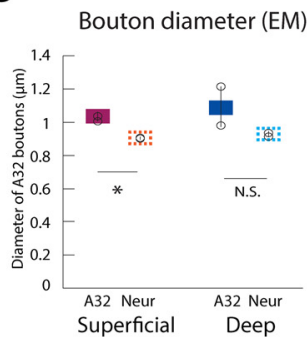

G

Volume of $A 32$ boutons in A25 (EM)

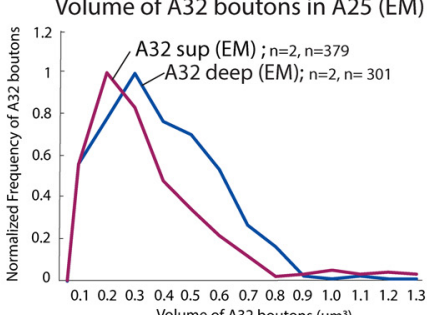

Volume of $\mathrm{A} 32$ boutons $\left(\mu \mathrm{m}^{3}\right)$

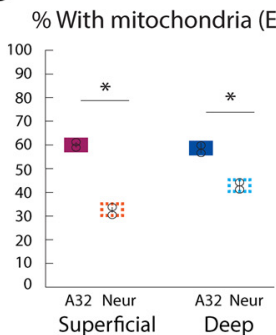

H

A32 bouton

volume (EM)

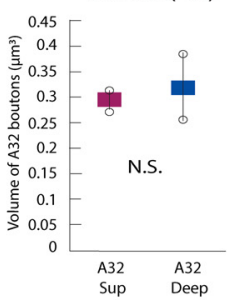

J

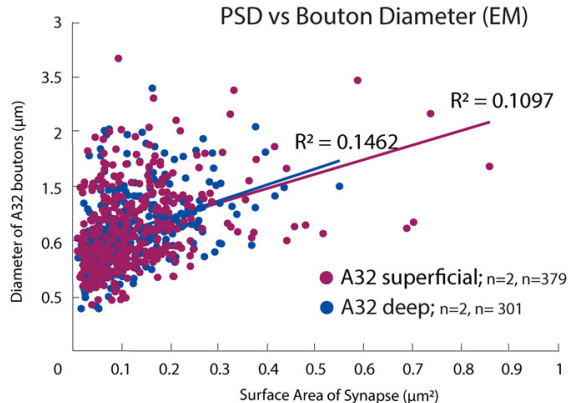

Figure 9. Structural characteristics of A32 terminals and their postsynaptic targets in A25 using EM. $A$, The superficial layers of A25, double-immunolabeled for A32 axonal processes (DAB, uniform dark precipitate), and PV (gold, black quanta, sometimes clustered). An A32 axonal termination (brown) forms synapses with three spines (sp), shaded in red, none of which contains $\mathrm{PV}^{+}$gold labeling. The A32 axonal termination formed a perforated synapse (ps) with one of these spines. Structures shaded in blue represent unlabeled axon terminations in the surrounding ROl that form asymmetric synapses, which are presumed excitatory and were used for neuropil analysis. Green shading represents postsynaptic structures of presumed excitatory boutons in the neuropil. B, Bouton diameters of labeled A32 terminations, obtained using BM and EM, and of terminations forming asymmetric synapses in the surrounding neuropil (dashed lines) in the superficial and deep layers of A25. C, Comparison of the average bouton diameter of A32 terminations and asymmetric synapses in the surrounding neuropil, in the superficial and deep layers of A25. D, Comparison of the proportion of A32 axonal terminations containing mitochondria with the proportion of terminations forming asymmetric synapses in the neuropil that contained mitochondria, in the superficial and deep layers of A25. $\boldsymbol{E}$, The proportion of A32 axonal terminations that formed perforated asymmetric synapses in comparison with synapses in the surrounding neuropil, in the superficial and deep layers of A25. $\boldsymbol{F}$, Proportion of A32 axonal terminations forming synapses on spines that contained a spine apparatus in comparison with the surrounding neuropil, in the superficial and deep layers of A25. G, Distribution of A32 bouton volumes in the superficial and deep layers of A25 for all 3D reconstructed A32 terminations as measured in serial sections. $\boldsymbol{H}$, Average bouton volume of A32 terminations in the superficial and deep layers of A25. I, PSD plotted against bouton volume for all 3D reconstructed A32 terminations. J, PSD plotted against bouton diameter for all 3D reconstructed A32 terminations. Data are mean \pm SD. Black circles represent individual values. ${ }^{*} p=0.05$. $b, B$ Bouton; $d$, dendrite; $m$, mitochondria; Neur, neuropil; ps, perforated synapse; sa, spine apparatus; sp, spine. 
A

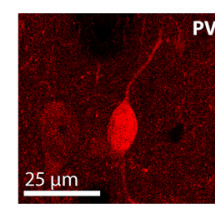

A25 superficial
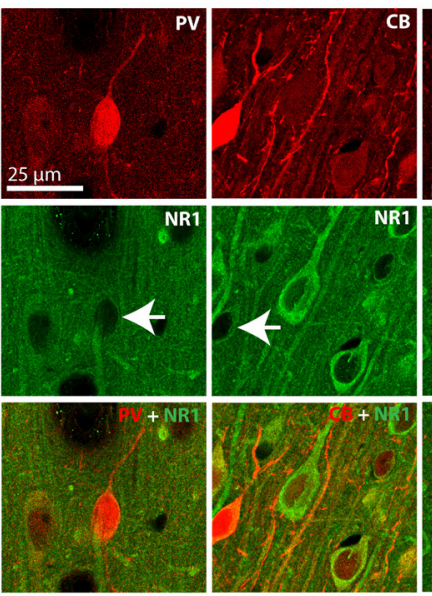

B

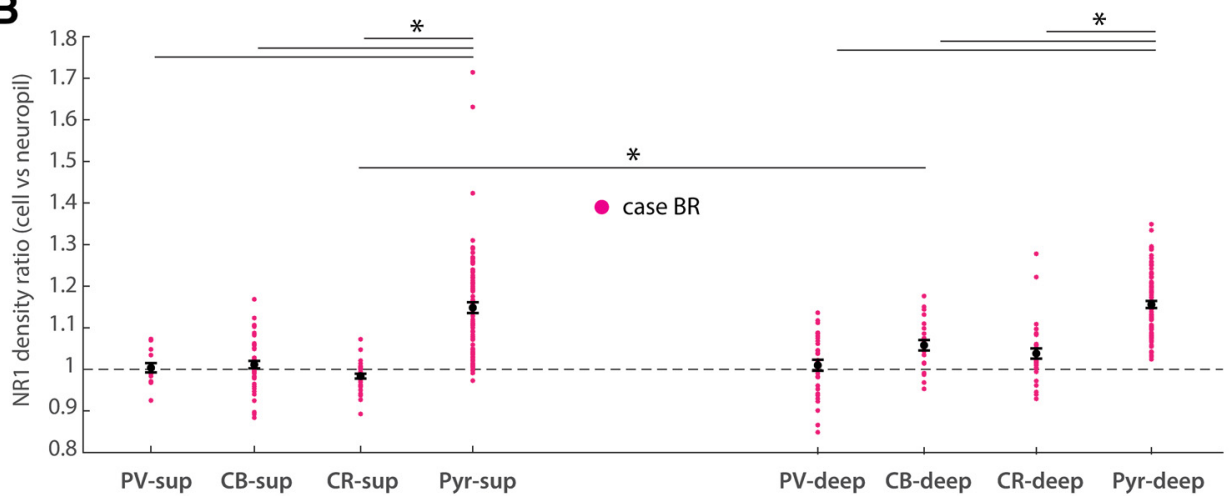

C

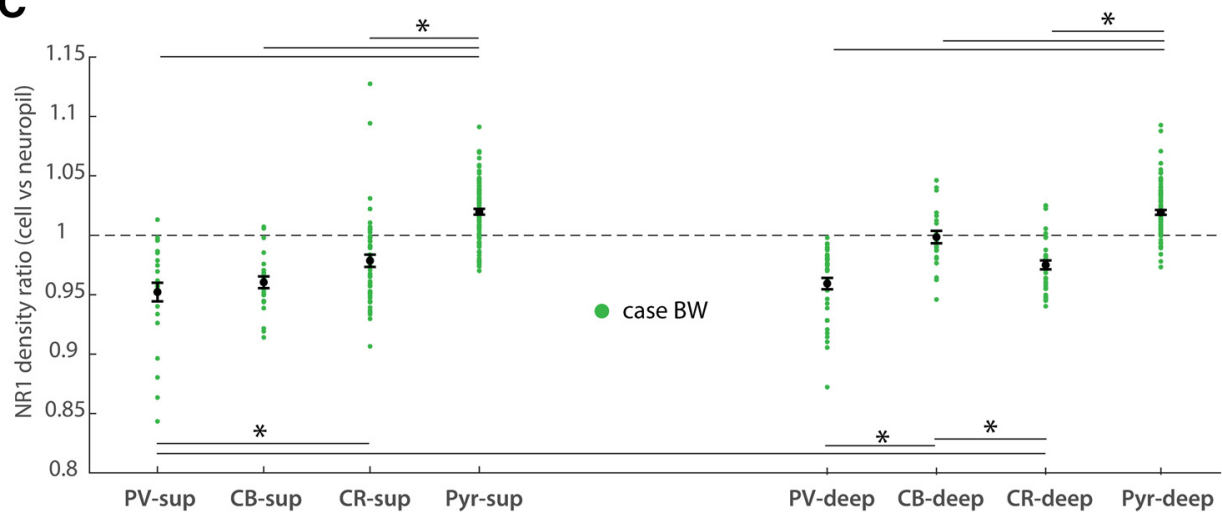

Figure 10. NR1 colocalization with PV, CB, and CR neurons in A25. A, Single focal planes obtained by CM show FOVs in superficial (left) and deep (right) layers of A25. FOVs are double-labeled using immunofluorescence for PV, CB, or CR (top row, red), and NR1 (middle row, green), the obligatory NMDAR subunit. Bottom row, Merged channels for CBP ${ }^{+}$and NR1 labeling. White arrows point to $\mathrm{CBP}^{+}$-labeled inhibitory cell bodies. Surrounding pyramidal neurons are rich in NMDARs. B, C, Quantification of NR1 labeling across presumed pyramidal (excitatory) and presumed inhibitory $\mathrm{CBP}^{+}$cell bodies in 2 cases (B, Case BR; $\boldsymbol{C}$, Case BW). Colored dots represent NR1 density ratio using all cells (of respective type) in an individual image. Black dots represent average \pm SEM over all images. Segmentation masks were prepared to outline $\mathrm{CBP}^{+}$cell bodies and pyramidal-shaped, presumed excitatory cell bodies. For $\mathrm{CB}$ neurons, only those that were morphologically consistent with inhibitory neurons and that featured dark $\mathrm{CB}^{+}$labeling were used. Segmentation masks were also prepared for sections of the neuropil, as a control for normalization within each image. This produced three masks for each image: one for pyramidal cell bodies, one for $\mathrm{CBP}^{+}$cell bodies (PV, CB, or (R), and one for neuropil regions. For each image, NR1 labeling intensity was computed as a density (over area) within each mask. The ratio of NR1 density in cells versus the neuropil in each image was then compared across (BP ${ }^{+}$ neurons and pyramidal neurons in superficial and deep layers of $A 25 .{ }^{*} p<0.05$.

terminations forming asymmetric (presumed excitatory) synapses in the neuropil of A25. We found that A32 boutons were larger than those in the neuropil in the superficial layers (Fig. 9 C; all layers: A32 $1.03 \pm 0.02 \mu \mathrm{m}$, neuropil $0.93 \pm 0.01 \mu \mathrm{m}$, one-way ANOVA, $F_{(1,14)}=6.89, p=0.02$; superficial: A32 $1.04 \pm 0.02 \mu \mathrm{m}$, neuropil $0.91 \pm 0.002 \mu \mathrm{m}$; one-way ANOVA, $F_{(1,2)}=18.813$, $p=0.049$ ), but not in the deep layers (Fig. 9C; A32 $1.09 \pm$ $0.12 \mu \mathrm{m}$, neuropil $0.94 \pm 0.01 \mu \mathrm{m}$, one-way ANOVA, $F_{(1,2)}=$ $1.507, p=0.344)$.

Other factors also affect synaptic efficacy, such as the presence of mitochondria in presynaptic terminations, the PSD surface area, and the presence of a perforated PSD (Stevens, 2004; 
A10/DLPFC

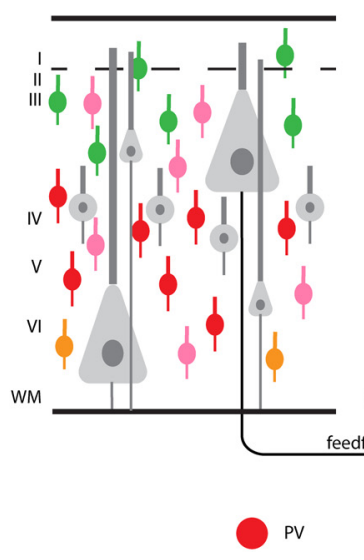

A32

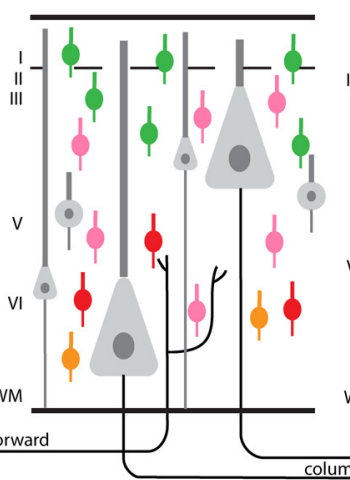

A25

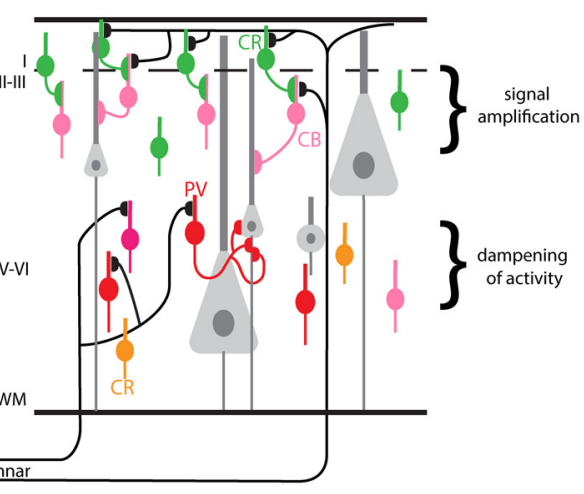

$\mathrm{CB}$

CR - inhibitory

CR - disinhibitory

Figure 11. Summary of findings. Superficial layer neurons from the dIPFC send feedforward projections to the deep layers of $A 32$. A32 sends projections to A25 originating in superficial and deep layers of A32. In the superficial layers of A25, A32 axonal terminations form synapses with CR (green) followed by CB (pink) neurons in the superficial layers. In the deep layers of A25, A32 axon terminals form synapses with PV (red) neurons, followed by CR (orange) and CB (pink) neurons. CR neurons (green) are thought to be largely disinhibitory in primate superficial cortical layers through synapses on surrounding inhibitory neurons. By predominantly targeting disinhibitory neurons in the superficial layers, pathways from A32 to the superficial layers of A25 may allow excitatory signals to propagate through the local circuitry. In the deep layers of $A 25, C R$ neurons are thought to have a net inhibitory effect. By predominantly targeting PV neurons in the deep layers, A32 engages a stronger inhibitory system and likely has a stronger ability to dampen activity in the local circuitry.

Bourne and Harris, 2008; Medalla and Luebke, 2015). The PSD surface area increases when synapses are perforated (Geinisman, 1993; Medalla and Luebke, 2015), and it is correlated with the density of AMPA receptors (Nava et al., 2014) and thus the magnitude of response in the postsynaptic neuron. We found that almost double the number of A32 terminations in the superficial and deep layers of A25 contained mitochondria compared with similar terminations in the surrounding neuropil (Fig. $9 D$; superficial, one-way ANOVA, $F_{(1,6)}=12.04, p=0.013$; deep, one-way ANOVA, $\left.F_{(1,6)}=11.591, p=0.014\right)$. A32 terminations also formed perforated synapses at a higher frequency than other presumed excitatory terminations in the neuropil (Fig. $9 E$, superficial, one-way ANOVA, $F_{(1,6)}=22.048, p=0.003$; deep, one-way ANOVA, $\left.F_{(1,6)}=22.287, p=0.003\right)$.

The spine apparatus is a specialized endoplasmic reticulum compartment in the spine head that is associated with plasticity, calcium dynamics, and second messenger systems (Jedlicka et al., 2008; Cugno et al., 2019; for review, see Segal et al., 2010). We found that the A32 pathway formed synapses on more spines containing a spine apparatus than terminations in the surrounding neuropil (Fig. 9F, A32 vs neuropil, superficial, one-way ANOVA, $F_{(1,6)}=18.457, p=0.005$; A32 vs neuropil, deep, oneway ANOVA, $\left.F_{(1,6)}=17.49, p=0.006\right)$. Collectively, these structural features suggest an increased efficacy of A32 terminations to propagate signals compared with other presumed excitatory synapses in the surrounding neuropil.

Figure $9 G, H$ shows the distribution and average volume of A32 axonal terminations in A25 $\left(0.29 \pm 0.02 \mu \mathrm{m}^{3}\right.$, superficial; $0.32 \pm 0.07 \mu \mathrm{m}^{3}$, deep, one-way ANOVA, $\left.F_{(1,2)}=0.126, p=0.76\right)$. Bouton volumes are within the range found for other corticocortical connections (Medalla et al., 2007; Medalla and Barbas, 2009).

We then investigated the relationship between PSD surface area and bouton size. In both superficial and deep layers, PSD surface area was significantly correlated with bouton volume (Fig. 9I) and bouton diameter (Fig. 9J), consistent with findings in other cortical pathways (Medalla et al., 2007; Medalla and Barbas, 2009; Bunce and Barbas, 2011).

\section{Distribution of NR1 receptors in area 25}

Finally, we examined the distribution of the NMDAR on $\mathrm{CBP}^{+}$ presumed inhibitory neurons and surrounding pyramidal- shaped excitatory neurons (Fig. 10) in 2 cases, using double immunofluorescence labeling for the CBPs and NR1, the obligatory subunit present in NMDARs (Fig. 10A). Results were similar across the 2 cases. Compared with the neuropil, pyramidal neurons in all layers were enriched in NMDARs (Fig. 10B,C; Case BR: one-way ANOVA $F_{(7,350)}=34.06 p=0$; Case BW: one-way ANOVA $\left.F_{(7,396)}=53.23, p=0\right)$. A moderate level of NR1 labeling was evident around the cytoplasm of the inhibitory neurons as well (Fig. 10B,C). In summary, our analysis showed that pyramidal neurons were more densely populated by NR1, and that CB and CR inhibitory neurons may contain denser NR1 populations than PV inhibitory neurons.

\section{Discussion}

We found that serial pathways link the dlPFC with A25, despite the weak direct connections between them. Moreover, the serial pathways showed laminar-specific patterns that suggest sequential flow of signals from the dlPFC to A32, which in turn innervates all layers of A25. These laminar-specific connections are consistent with predictions of the Structural Model (Barbas and Rempel-Clower, 1997; for review, see Barbas, 2015; GarcíaCabezas et al., 2019).

A rich complement to understanding the laminar patterns of cortical connectivity is the ability to predict the interface of pathways with laminar-specific local inhibitory neurons. Long-range corticocortical pathways mostly innervate excitatory postsynaptic targets at $\sim 75 \%-80 \%$ in primates (for review, see Barbas and Zikopoulos, 2007; Medalla and Barbas, 2014; Anderson et al., 2015; Barbas, 2015; Barbas and García-Cabezas, 2016; Barbas et al., 2018). However, the smaller but significant complement of inhibitory postsynaptic targets of cortical pathways ( 20\%-25\%) likely has a powerful effect on local firing patterns (Dehghani et al., 2016; Le Van Quyen et al., 2016; Telenczuk et al., 2017).

Differences in inhibitory neuron composition within the cortical column and across cortical areas shown here and elsewhere (Dombrowski et al., 2001; Medalla et al., 2017) may significantly contribute to the remarkable behavioral flexibility in primates (Markram et al., 2004; Isaacson and Scanziani, 2011; Marin, 2012). Graded variations in the density of inhibitory neurons 


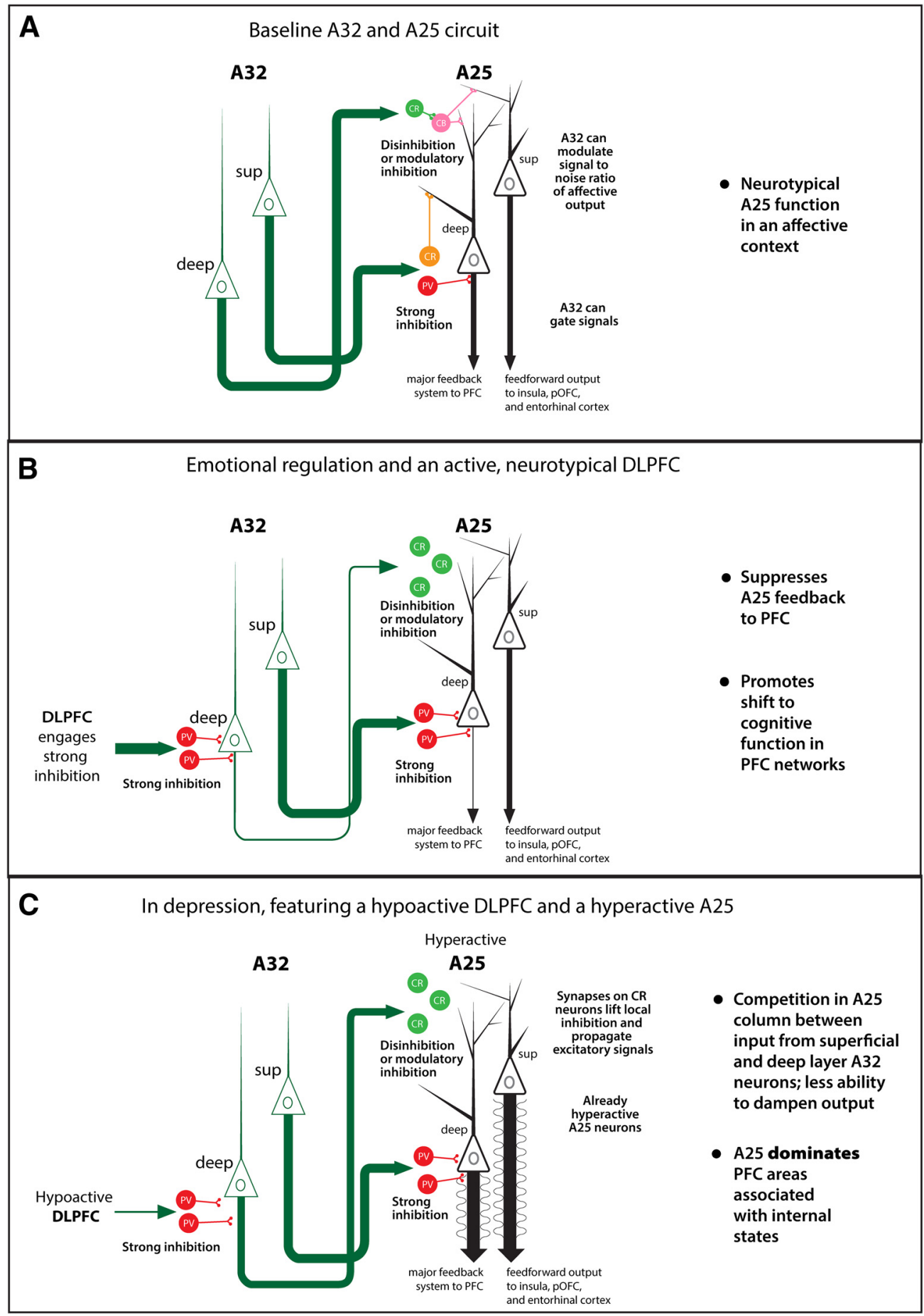

Figure 12. Summary circuit schematic of serial pathway from dIPFC to $A 32$ to $A 25$ for various functional states. $A, A$ circuit model of the synaptic interactions from $A 32$ to $A 25$ based on the present findings. The model leaves out other contributions to cortical microcircuitry (e.g., neuromodulatory and thalamocortical input), is unidirectional according to the serial pathway under study, and thus does not reflect reciprocal connections. Green represents neurons with excitatory effects. Shades of red represent neurons with inhibitory effects on the circuit. In the superficial layers of A25, terminations from A32 modulate the signal-to-noise ratio through synapses with the disinhibitory CR (light green) and modulatory CB (pink) inhibitory microsystems. In the deep layers of A25, terminations from A32 can gate signals through the powerful PV (red) and inhibitory CR (orange) microsystem. $\boldsymbol{B}$, A putative model of neurotypical emotional regulation, based on the patterns of projections from the dIPFC to A32 to A25 and physiologic studies. For simplicity, the inhibitory microsystems illustrated in $\boldsymbol{A}$ have been collapsed to CR in the superficial layers and PV in the deep layers because they are the primary inhibitory targets of A32 in A25. The dIPFC and A32 are necessary for emotional regulation as summarized in functional studies. dIPFC terminations in A32 may suppress the deep layers of A32 through the strong PV inhibitory microsystem, reducing output to the superficial layers of A25. This leaves the superficial layers of A32 active, which engage the strong PV inhibitory microsystem in the deep layers of A25, dampening the contribution of A25 to ongoing processes in the PFC. Deep layer neurons of A25 project to many PFC areas that contribute to internal state (Joyce and Barbas, 2018); and when that output is suppressed, resources can be focused elsewhere as needed. C, A putative model in depression. Metabolic A25 activity is heightened, according to reported functional pathology in depression. Here, those findings have been interpreted to mean that individual neurons are in a more active state than in a neurotypical A25 (indicated by sinusoidal waves around axons). Functional pathology in depression also involves a hypoactive dIPFC. An attenuated influence of the dIPFC on A32 predicts that both the superficial and deep layers of A32 exert influence on A25. In the superficial layers of A25, lifted inhibition by A32 terminations on CR neurons allows local excitatory signals to propagate unfettered and potentially exacerbates the already hyperactive A25 neurons. In the deep layers, terminations from A32 may not exert enough inhibition to 
engaged within cortical networks may contribute to transient changes in synchrony (Bonnefond et al., 2017). Variations in inhibitory neuron density can also help explain functional specializations across cortical areas. For example, the elevated density of PV neurons in the middle-to-deep layers of A46 allows extensive lateral inhibition among layer IIIc pyramidal ensembles that refine mental representations in working memory (GoldmanRakic, 1995; for review, see Arnsten, 2013), ultimately contributing to the dlPFC's important role in cognition.

The inverse relationship in functional activity between the dlPFC and A25 suggests specific circuit mechanisms involving inhibitory neurons. However, because direct connections between the dIPFC and A25 are sparse, the mechanism is more complex. A32 is a hub that links functionally disparate prefrontal areas (Barbas et al., 1999; Tang et al., 2019), and participates in cognitive, attentional, affective, and default mode networks (Bush et al., 2000; Inzlicht et al., 2015; Buckner and DiNicola, 2019). It thus has a part in wide-ranging functions and is positioned to balance diverse cortical processes.

\section{The serial pathway: A32 connects A25 with the dlPFC and A10}

The circuit revealed here has mechanistic implications for understanding how cognitive information, often associated with the dlPFC, can be integrated with affective information processing in A25, as summarized in Figure 11. In A32, dlPFC and A10 pathways terminate mostly in the deep layers, but both superficial and deep layer A32 neurons project to A25. The influence of dlPFC on A25 activity thus may be weighted more heavily through its denser projections to the deep A32 layers.

Our circuit findings have distinct implications when viewed in the context of their interactions with inhibitory neurons. In the superficial layers of A25, A32 terminations formed synapses on spines of excitatory neurons, and with CR neurons, which are thought to be disinhibitory because they target nearby inhibitory neurons (Meskenaite, 1997; Melchitzky, et al., 2005; for review, see Barbas et al., 2018). A32 axonal terminations also innervate some CB inhibitory processes in the superficial layers. Inhibitory $\mathrm{CB}$ neurons are thought to be modulatory via their mid-to-distal dendritic targeting of nearby pyramidal neurons (DeFelipe, 1997; Wang et al., 2004). Much about the function of these inhibitory systems in primates is unknown, but computational modeling suggests that the $\mathrm{CB}$ superficial inhibitory microsystem may be suited to tune the local signal-to-noise ratio (Constantinidis et al., 2002; Wang et al., 2004). We suggest that, in the superficial layers, A32 may boost relevant incoming excitatory signals from diverse sources via the CR system, while dampening irrelevant signals via the $\mathrm{CB}$ system.

On the other hand, A32 terminations in the deep layers of A25 interact more heavily with PV inhibitory neurons, where they are more abundant than in the superficial layers. PV inhibitory neurons can exercise strong perisomatic inhibition of nearby pyramidal neurons (DeFelipe, 1997; Mikkonen et al., 1997) and are fast-spiking (Zaitsev et al., 2009). CR neurons in the deep layers are thought to have a net inhibitory effect, at least in the primary visual cortex (Meskenaite, 1997). Notably, it is the deep layer neurons in A25 that project to other prefrontal areas,

dampen A25 output. In short, A25 neurons are hyperactive, and A32 cannot suppress the abnormally high activity. A25 output to other PFC areas that are associated with internal states is exaggerated (thick arrows). pOFC, Posterior orbitofrontal cortex; sup, superficial. as the ultimate feedback system (Joyce and Barbas, 2018). The powerful inhibitory system that A32 engages in the deep layers of A25 likely has direct impact on this feedback system. Deep layer neurons in A25 also project to subcortical sites, such as the amygdala, hypothalamus, and ventral striatum, which support its role in homeostasis (Ongur et al., 1998; Rempel-Clower and Barbas, 1998; Barbas et al., 2003; Ghashghaei et al., 2007; Heilbronner et al., 2016). Thus, A32 terminations in the deep layers of A25 may be a circuit mechanism to dampen affect-associated A25 activity within prefrontal networks and downstream autonomic projections (Kaada et al., 1949; Wallis et al., 2017; Lacuey et al., 2018; Alexander et al., 2019b; Zeredo et al., 2019; for review, see Alexander et al., 2019a), to allow a switch to cognitive tasks based on context.

The functional implications of the pathway from A32 to A25 are strengthened by several of its synaptic features compared with unlabeled excitatory synapses in the surrounding neuropil in A25. First, A32 boutons were larger, suggesting a higher input weight on local neurons (Germuska et al., 2006). Second, A32 terminations were more likely to contain mitochondria, which are characteristic of active synapses (for review, see Thomson, 2000; Devine and Kittler, 2018). And third, A32 boutons formed more perforated synapses in A25, which increase the surface area and the number of postsynaptic excitatory receptors (Geinisman, 1993; Nava et al., 2014; Medalla and Luebke, 2015). Together, these findings imply that terminations from the frontal A32 hub (Barbas et al., 1999; Tang et al., 2019) have a pronounced input weight in A25. There is evidence of rostrocaudal and dorsoventral axes in neuronal encoding of valence in A25 (Monosov and Hikosaka, 2012). Our study demonstrates that A32 has access to all subregions of A25, suggesting flexible recruitment of neuronal ensembles based on context.

Figure 12 shows a proposed model of the sequential pathway interactions, beginning with the laminar connections from A32 to A25 (Fig. 12A). As shown in Figure 12B, when all the pieces of the serial pathway are combined, the circuit logic predicts that superficial layer dlPFC neurons can dampen the deep layers of A25, which constitute the major output to most PFC. We speculate that this is a critical mechanism to shift toward a cognitive focus in prefrontal networks, because dampening the deep layers of A25 would decrease their feedback input to other PFC areas associated with emotion and internal state (Joyce and Barbas, 2018). Together, the model illustrates a circuit mechanism for prefrontal emotional regulation, in accord with functional evidence in neurotypical humans (Sinha et al., 2016).

\section{Differential effects of the direct A10 pathway and the serial pathway through A32}

A10 has indirect access to A25 through A32 and a moderate direct pathway to A25 (Joyce and Barbas, 2018). A10 is broadly associated with complex cognition (Koechlin et al., 1999; Semendeferi et al., 2001; Dreher et al., 2008; Medalla and Barbas, 2010, 2014) and emotional regulation (Beauregard et al., 2001; Ochsner et al., 2004; Blair et al., 2007). Recent findings have suggested that both A10 and the dlPFC modulate the shift between affective- and cognitive-related networks through subgenual activity (Ramirez-Mahaluf et al., 2018; for review, see Rive et al., 2013). The monosynaptic pathway from A10 to A25 is feedforward-biased (Joyce and Barbas, 2018), and thus may act as a stronger and faster mechanism to dampen A25 activity than the multisynaptic sequential pathway through A32. The dual circuit mechanisms from A10 to A25 is consistent with the greater emotional flexibility in primates (Semendeferi et al., 2011). 


\section{Implications for mood dysregulation}

The Structural Model has recently been validated to predict the connectional pathology of complex psychiatric disorders in humans (Zikopoulos et al., 2018). Mood disorders involve network dysfunction among many distributed brain areas (Mayberg, 1997; Drysdale et al., 2017). However, the prefrontal areas studied here are consistently implicated (Mayberg, 1997; Mayberg et al., 1999; Riva-Posse et al., 2014; Wang et al., 2016; Ramirez-Mahaluf et al., 2017; for review, see Rive et al., 2013). Inverse functional correlation has been associated between the metabolic activity of dPFC and subgenual cortex in depression (Mayberg et al., 1999, 2005). Significantly, many studies have cited pretreatment metabolism of A32 as a predictor of remission (Pizzagalli et al., 2001; Davidson et al., 2003; Seminowicz et al., 2004; Salvadore et al., 2009; for review, see DeRubeis et al., 2008; Waters and Mayberg, 2017). This evidence implies that the reliability of the serial pathway is crucial for emotional equilibrium. Figure $12 \mathrm{C}$ illustrates the predicted circuit mechanism when dlPFC activity is attenuated, leading to inability to dampen A25 activity in depression.

Runaway activity in A25 may lead to iterative drive of the default mode network in depression (Berman et al., 2011; Whitfield-Gabrieli and Ford, 2012; Hamilton et al., 2015), a state correlated with rumination and excessive negatively valenced self-directed processes. The output of A25 is heavily weighted toward other prefrontal areas involved in emotion and internal states (Joyce and Barbas, 2018). A robust serial pathway from dlPFC may dampen excessive activity in A25 and prevent perseverative cycling within the network. There is evidence that repetitive transcranial magnetic stimulation in the dlPFC modulates connectivity of the subgenual cingulate with the default mode network (Liston et al., 2014), and produces antidepressant effects (Fox et al., 2012; Weigand et al., 2018; Hadas et al., 2019; for review, see Gaynes et al., 2014; Trevizol and Blumberger, 2019). Unsuccessful repetitive transcranial magnetic stimulation attempts may reflect a weakened serial pathway, suggesting that invasive methods, such as deep brain stimulation, may be needed to reduce activity in A25 in depression (Mayberg et al., 2005, 2009; for review, see Drobisz and Damborska, 2019; La Torre et al., 2020).

We also found rich populations of NMDARs on pyramidal neurons in A25, which are also the densest among PFC areas in humans (Palomero-Gallagher et al., 2008; Palomero-Gallagher et al., 2009). One possible way to dampen A25 hyperactivity could be through NMDAR antagonism, which may contribute to ketamine's rapid antidepressant effects in humans. In nonhuman primates, ketamine has preferential network plasticity effects ( $\mathrm{Lv}$ et al., 2016) and antidepressant-promoting effects (Alexander et al., 2019b) mediated by A25. Ketamine administration to the infralimbic-cortex in rodents also elicits antidepressant-promoting effects (Fuchikami et al., 2015; Ferenczi et al., 2016; ModaSava et al., 2019), although rodent-primate homologies are unresolved and beyond the scope of this study.

Evidence implicates inhibitory neurons in depression as well (for review, see Luscher et al., 2011; Luscher and Fuchs, 2015), which express NMDARs differentially across types of inhibitory neurons and areas (Huntley et al., 1997; Gonzalez-Albo et al., 2001). We found that NMDARs were expressed on all inhibitory neurons in A25. However, among inhibitory classes, CR neurons were most numerous in $\mathrm{A} 25$, and in humans $\mathrm{CR}$ populations have expanded in number and architectonic detail (del Rio and DeFelipe, 1997; Dzaja et al., 2014). Because of their disinhibitory nature, we speculate that the superficial layer CR system could be one intriguing possibility for the ultra-rapid effects of intranasal ketamine, which is thought to target A25 (Opler et al., 2016).

When the dlPFC is hypoactive in depression, input from A32 that can dampen deep layer activity in A25 is attenuated (Fig. $12 C$ ), leaving A32 inputs in the upper and deep layers in competition. A driving force of ketamine's effect in A25 may be reduction in activity of pyramidal neurons (Wang et al., 2013) and the numerous superficial layer CR neurons. The binding of NMDAR antagonists on pyramidal neurons and superficial layer CR neurons in patients with depression may mimic the regulatory effects of the neurotypical serial pathway revealed here (Fig. $12 B)$.

\section{References}

Alexander L, Clarke HF, Roberts AC (2019a) A focus on the functions of area 25. Brain Sci 9:129.

Alexander L, Gaskin PL, Sawiak SJ, Fryer TD, Hong YT, Cockcroft GJ, Clarke HF, Roberts AC (2019b) Fractionating blunted reward processing characteristic of anhedonia by over-activating primate subgenual anterior cingulate cortex. Neuron 101:307-320.e306.

Arnsten AF (2013) The neurobiology of thought: the groundbreaking discoveries of Patricia Goldman-Rakic 1937-2003. Cereb Cortex 23:2269-2281.

Anderson MC, Bunce JG, Barbas H (2015) Prefrontal-hippocampal pathways underlying inhibitory control over memory. Neurobiol Learn Mem, 134 Pt A, 145-161.

Barbas H (1993) Organization of cortical afferent input to orbitofrontal areas in the rhesus monkey. Neuroscience 56:841-864.

Barbas H (2015) General cortical and special prefrontal connections: principles from structure to function. Annu Rev Neurosci 38:269-289.

Barbas H, Pandya DN (1989) Architecture and intrinsic connections of the prefrontal cortex in the rhesus monkey. J Comp Neurol 286:353-375.

Barbas H, Rempel-Clower N (1997) Cortical structure predicts the pattern of corticocortical connections. Cereb Cortex 7:635-646.

Barbas H, Ghashghaei H, Dombrowski SM, Rempel-Clower NL (1999) Medial prefrontal cortices are unified by common connections with superior temporal cortices and distinguished by input from memory-related areas in the rhesus monkey. J Comp Neurol 410:343-367.

Barbas H, Zikopoulos B (2007) The prefrontal cortex and flexible behavior. Neuroscientist 13:532-545.

Barbas H, García-Cabezas MA (2016) How the prefrontal executive got its stripes. Curr Opin Neurobiol 40:125-134

Barbas H, Saha S, Rempel-Clower N, Ghashghaei T (2003) Serial pathways from primate prefrontal cortex to autonomic areas may influence emotional expression. BMC Neurosci 4:25.

Barbas H, Wang J, Joyce MK, García-Cabezas MA (2018) Pathway mechanism for excitatory and inhibitory control in working memory. J Neurophysiol 120:2659-2678.

Beauregard M, Levesque J, Bourgouin P (2001) Neural correlates of conscious self-regulation of emotion. J Neurosci 21:RC165.

Berman MG, Peltier S, Nee DE, Kross E, Deldin PJ, Jonides J (2011) Depression, rumination and the default network. Soc Cogn Affect Neurosci 6:548-555.

Blair KS, Smith BW, Mitchell DG, Morton J, Vythilingam M, Pessoa L, Fridberg D, Zametkin A, Sturman D, Nelson EE, Drevets WC, Pine DS, Martin A, Blair RJ (2007) Modulation of emotion by cognition and cognition by emotion. Neuroimage 35:430-440.

Bonnefond M, Kastner S, Jensen O (2017) Communication between brain areas based on nested oscillations. eNeuro 4:ENEURO.0153-16.2017.

Bourne JN, Harris KM (2008) Balancing structure and function at hippocampal dendritic spines. Annu Rev Neurosci 31:47-67.

Buckner RL, DiNicola LM (2019) The brain's default network: updated anatomy, physiology and evolving insights. Nat Rev Neurosci 20:593-608.

Bunce JG, Barbas H (2011) Prefrontal pathways target excitatory and inhibitory systems in memory-related medial temporal cortices. Neuroimage 55:1461-1474.

Bunce JG, Zikopoulos B, Feinberg M, Barbas H (2013) Parallel prefrontal pathways reach distinct excitatory and inhibitory systems in memoryrelated rhinal cortices. J Comp Neurol 512:4260-4283. 
Bush G, Luu P, Posner MI (2000) Cognitive and emotional influences in anterior cingulate cortex. Trends Cogn Sci 4:215-222.

Constantinidis C, Williams GV, Goldman-Rakic PS (2002) A role for inhibition in shaping the temporal flow of information in prefrontal cortex. Nat Neurosci 5:175-180.

Cugno A, Bartol TM, Sejnowski TJ, Iyengar R, Rangamani P (2019) Geometric principles of second messenger dynamics in dendritic spines. Sci Rep 9:11676.

Datta D, Arnsten AF (2019) Loss of prefrontal cortical higher cognition with uncontrollable stress: molecular mechanisms, changes with age, and relevance to treatment. Brain Sci 9:113.

Davidson RJ, Irwin W, Anderle MJ, Kalin NH (2003) The neural substrates of affective processing in depressed patients treated with venlafaxine. Am J Psychiatry 160:64-75.

DeFelipe J (1997) Types of neurons, synaptic connections and chemical characteristics of cells immunoreactive for calbindin-D28K, parvalbumin and calretinin in the neocortex. J Chem Neuroanat 14:1-19.

Dehghani N, Peyrache A, Telenczuk B, Le Van Quyen M, Halgren E, Cash SS, Hatsopoulos NG, Destexhe A (2016) Dynamic balance of excitation and inhibition in human and monkey neocortex. Sci Rep 6:23176.

del Rio MR, DeFelipe J (1997) Synaptic connections of calretinin-immunoreactive neurons in the human neocortex. J Neurosci 17:5143-5154.

DeRubeis RJ, Siegle GJ, Hollon SD (2008) Cognitive therapy versus medication for depression: treatment outcomes and neural mechanisms. Nat Rev Neurosci 9:788-796.

Devine MJ, Kittler JT (2018) Mitochondria at the neuronal presynapse in health and disease. Nat Rev Neurosci 19:63-80.

Dombrowski SM, Barbas H (1996) Differential expression of NADPH diaphorase in functionally distinct prefrontal cortices in the rhesus monkey. Neuroscience 72:49-62.

Dombrowski SM, Hilgetag CC, Barbas H (2001) Quantitative architecture distinguishes prefrontal cortical systems in the rhesus monkey. Cereb Cortex 11:975-988.

Dreher JC, Koechlin E, Tierney M, Grafman J (2008) Damage to the frontopolar cortex is associated with impaired multitasking. PLoS One 3:e3227.

Drobisz D, Damborska A (2019) Deep brain stimulation targets for treating depression. Behav Brain Res 359:266-273.

Drysdale AT, Grosenick L, Downar J, Dunlop K, Mansouri F, Meng Y, Fetcho RN, Zebley B, Oathes DJ, Etkin A, Schatzberg AF, Sudheimer K, Keller J, Mayberg HS, Gunning FM, Alexopoulos GS, Fox MD, PascualLeone A, Voss HU, Casey BJ, et al. (2017) Resting-state connectivity biomarkers define neurophysiological subtypes of depression. Nat Med 23:28-38.

Dzaja D, Hladnik A, Bicanic I, Bakovic M, Petanjek Z (2014) Neocortical calretinin neurons in primates: increase in proportion and microcircuitry structure. Front Neuroanat 8:103

Ferenczi EA, Zalocusky KA, Liston C, Grosenick L, Warden MR, Amatya D, Katovich K, Mehta H, Patenaude B, Ramakrishnan C, Kalanithi P, Etkin A, Knutson B, Glover GH, Deisseroth K (2016) Prefrontal cortical regulation of brainwide circuit dynamics and reward-related behavior. Science 351:aac9698.

Fiala JC (2005) Reconstruct: a free editor for serial section microscopy. J Microsc 218:52-61.

Fox MD, Buckner RL, White MP, Greicius MD, Pascual-Leone A (2012) Efficacy of transcranial magnetic stimulation targets for depression is related to intrinsic functional connectivity with the subgenual cingulate. Biol Psychiatry 72:595-603.

Fuchikami M, Thomas A, Liu R, Wohleb ES, Land BB, DiLeone RJ, Aghajanian GK, Duman RS (2015) Optogenetic stimulation of infralimbic PFC reproduces ketamine's rapid and sustained antidepressant actions. Proc Natl Acad Sci USA 112:8106-8111.

Gabbott PL, Bacon SJ (1996a) Local circuit neurons in the medial prefrontal cortex (areas 24a,b,c, 25 and 32) in the monkey: I. Cell morphology and morphometrics. J Comp Neurol 364:567-608.

Gabbott PL, Bacon SJ (1996b) Local circuit neurons in the medial prefrontal cortex (areas 24a,b,c, 25 and 32) in the monkey: II. Quantitative areal and laminar distributions. J Comp Neurol 364:609-636.

García-Cabezas MA, Barbas H (2017) Anterior cingulate pathways may affect emotions through orbitofrontal cortex. Cereb Cortex 27:4891-4910.

García-Cabezas MA, John YJ, Barbas H, Zikopoulos B (2016) Distinction of neurons, glia and endothelial cells in the cerebral cortex: an algorithm based on cytological features. Front Neuroanat 10:107.
Garcia-Cabezas MA, Joyce MP, John Y, Zikopoulos B, Barbas H (2017) Mirror trends of plasticity and stability indicators in primate prefrontal cortex. Eur J Neurosci 46:2392-2405.

García-Cabezas MA, Zikopoulos B, Barbas H (2019) The structural model: a theory linking connections, plasticity, pathology, development and evolution of the cerebral cortex. Brain Struct Funct 224:985-1008.

Gaynes BN, Lloyd SW, Lux L, Gartlehner G, Hansen RA, Brode S, Jonas DE, Swinson Evans T, Viswanathan M, Lohr KN (2014) Repetitive transcranial magnetic stimulation for treatment-resistant depression: a systematic review and meta-analysis. J Clin Psychiatry 75:477-489.quiz 489.

Geinisman Y (1993) Perforated axospinous synapses with multiple, completely partitioned transmission zones: probable structural intermediates in synaptic plasticity. Hippocampus 3:417-433.

Germuska M, Saha S, Fiala J, Barbas H (2006) Synaptic distinction of laminar-specific prefrontal-temporal pathways in primates. Cereb Cortex 16:865-875.

Ghashghaei HT, Hilgetag CC, Barbas H (2007) Sequence of information processing for emotions based on the anatomic dialogue between prefrontal cortex and amygdala. Neuroimage 34:905-923.

Goldman-Rakic PS (1995) Cellular basis of working memory. Neuron 14:477-485

Gonzalez-Albo MC, Elston GN, DeFelipe J (2001) The human temporal cortex: characterization of neurons expressing nitric oxide synthase, neuropeptides and calcium-binding proteins, and their glutamate receptor subunit profiles. Cereb Cortex 11:1170-1181.

Gould TD, Zarate CA Jr, Thompson SM (2019) Molecular pharmacology and neurobiology of rapid-acting antidepressants. Annu Rev Pharmacol Toxicol 59:213-236.

Gundersen HJ (1986) Stereology of arbitrary particles: a review of unbiased number and size estimators and the presentation of some new ones, in memory of William R. Thompson. J Microsc 143: 3-45.

Hadas I, Sun Y, Lioumis P, Zomorrodi R, Jones B, Voineskos D, Downar J, Fitzgerald PB, Blumberger DM, Daskalakis ZJ (2019) Association of repetitive transcranial magnetic stimulation treatment with subgenual cingulate hyperactivity in patients with major depressive disorder: a secondary analysis of a randomized clinical trial. JAMA Netw Open 2: e195578.

Hamilton JP, Farmer M, Fogelman P, Gotlib IH (2015) Depressive rumination, the default-mode network, and the dark matter of clinical neuroscience. Biol Psychiatry 78:224-230.

Heilbronner SR, Rodriguez-Romaguera J, Quirk GJ, Groenewegen HJ, Haber SN (2016) Circuit-based corticostriatal homologies between rat and primate. Biol Psychiatry 80:509-521.

Howard CV, Reed MG (1998) Unbiased stereology, three-dimensional measurement in microscopy. Oxford: BIOS Scientific.

Huntley GW, Vickers JC, Morrison JH (1997) Quantitative localization of NMDAR1 receptor subunit immunoreactivity in inferotemporal and prefrontal association cortices of monkey and human. Brain Res 749:245262.

Inzlicht M, Bartholow BD, Hirsh JB (2015) Emotional foundations of cognitive control. Trends Cogn Sci 19:126-132.

Isaacson JS, Scanziani M (2011) How inhibition shapes cortical activity. Neuron 72:231-243.

Jedlicka P, Vlachos A, Schwarzacher SW, Deller T (2008) A role for the spine apparatus in LTP and spatial learning. Behav Brain Res 192:12-19.

Joyce MP, Barbas H (2018) Cortical connections position primate area 25 as a keystone for interoception, emotion, and memory. J Neurosci 38:16771698.

Kaada BR, Pribram KH, Epstein JA (1949) Respiratory and vascular responses in monkeys from temporal pole, insula, orbital surface and cingulate gyrus. J Neurophysiol 12:348-356.

Koechlin E, Basso G, Pietrini P, Panzer S, Grafman J (1999) The role of the anterior prefrontal cortex in human cognition. Nature 399:148-151.

La Torre D, Della Torre A, Chirchiglia D, Volpentesta G, Guzzi G, Lavano A (2020) Deep brain stimulation for treatment-resistant depression: a safe and effective option. Expert Rev Neurother 20:449-457.

Lacuey N, Hampson JP, Theeranaew W, Zonjy B, Vithala A, Hupp NJ, Loparo KA, Miller JP, Lhatoo SD (2018) Cortical structures associated with human blood pressure control. JAMA Neurol 75:194-202.

Le Van Quyen M, Muller LE 2nd, Telenczuk B, Halgren E, Cash S, Hatsopoulos NG, Dehghani N, Destexhe A (2016) High-frequency 
oscillations in human and monkey neocortex during the wake-sleep cycle. Proc Natl Acad Sci USA 113:9363-9368.

Liston C, Chen AC, Zebley BD, Drysdale AT, Gordon R, Leuchter B, Voss HU, Casey BJ, Etkin A, Dubin MJ (2014) Default mode network mechanisms of transcranial magnetic stimulation in depression. Biol Psychiatry 76:517-526.

Luscher B, Fuchs T (2015) GABAergic control of depression-related brain states. Adv Pharmacol 73:97-144.

Luscher B, Shen Q, Sahir N (2011) The GABAergic deficit hypothesis of major depressive disorder. Mol Psychiatry 16:383-406.

Lv Q, Yang L, Li G, Wang Z, Shen Z, Yu W, Jiang Q, Hou B, Pu J, Hu H, Wang Z (2016) Large-scale persistent network reconfiguration induced by ketamine in anesthetized monkeys: relevance to mood disorders. Biol Psychiatry 79:765-775.

Marin O (2012) Interneuron dysfunction in psychiatric disorders. Nat Rev Neurosci 13:107-120.

Markram H, Toledo-Rodriguez M, Wang Y, Gupta A, Silberberg G, Wu C (2004) Interneurons of the neocortical inhibitory system. Nat Rev Neurosci 5:793-807.

Mayberg HS (1997) Limbic-cortical dysregulation: a proposed model of depression. J Neuropsychiatry Clin Neurosci 9:471-481.

Mayberg HS (2003) Modulating dysfunctional limbic-cortical circuits in depression: towards development of brain-based algorithms for diagnosis and optimised treatment. Br Med Bull 65:193-207.

Mayberg HS (2009) Targeted electrode-based modulation of neural circuits for depression. J Clin Invest 119:717-725.

Mayberg HS, Liotti M, Brannan SK, McGinnis S, Mahurin RK, Jerabek PA, Silva JA, Tekell JL, Martin CC, Lancaster JL, Fox PT (1999) Reciprocal limbic-cortical function and negative mood: converging PET findings in depression and normal sadness. Am J Psychiatry 156:675-682.

Mayberg HS, Lozano AM, Voon V, McNeely HE, Seminowicz D, Hamani C, Schwalb JM, Kennedy SH (2005) Deep brain stimulation for treatmentresistant depression. Neuron 45:651-660.

Medalla M, Barbas H (2009) Synapses with inhibitory neurons differentiate anterior cingulate from dorsolateral prefrontal pathways associated with cognitive control. Neuron 61:609-620.

Medalla M, Barbas H (2010) Anterior cingulate synapses in prefrontal areas 10 and 46 suggest differential influence in cognitive control. J Neurosci 30:16068-16081.

Medalla M, Barbas H (2014) Specialized prefrontal "auditory fields": organization of primate prefrontal-temporal pathways. Front Neurosci 8:77.

Medalla M, Luebke JI (2015) Diversity of glutamatergic synaptic strength in lateral prefrontal versus primary visual cortices in the rhesus monkey. J Neurosci 35:112-127.

Medalla M, Lera P, Feinberg M, Barbas H (2007) Specificity in inhibitory systems associated with prefrontal pathways to temporal cortex in primates. Cereb Cortex 17:i136-i150.

Medalla M, Gilman JP, Wang JY, Luebke JI (2017) Strength and diversity of inhibitory signaling differentiates primate anterior cingulate from lateral prefrontal cortex. J Neurosci 37:4717-4734.

Melchitzky DS, Eggan SM, Lewis DA (2005) Synaptic targets of calretinincontaining axon terminals in macaque monkey prefrontal cortex. Neuroscience 130:185-195

Meskenaite V (1997) Calretinin-immunoreactive local circuit neurons in area 17 of the cynomolgus monkey, Macaca fascicularis. J Comp Neurol 379:113-132.

Mikkonen M, Soininen H, Pitkänen A (1997) Distribution of parvalbumin-, calretinin-, and calbindin-D28k-immunoreactive neurons and fibers in the human entorhinal cortex. J Comp Neurol 388:64-88.

Moda-Sava RN, Murdock MH, Parekh PK, Fetcho RN, Huang BS, Huynh TN, Witztum J, Shaver DC, Rosenthal DL, Alway EJ, Lopez K, Meng Y, Nellissen L, Grosenick L, Milner TA, Deisseroth K, Bito H, Kasai H, Liston C (2019) Sustained rescue of prefrontal circuit dysfunction by antidepressant-induced spine formation. Science 364:eaat80784.

Monosov IE, Hikosaka O (2012) Regionally distinct processing of rewards and punishments by the primate ventromedial prefrontal cortex. J Neurosci 32:10318-10330.

Nava N, Chen F, Wegener G, Popoli M, Nyengaard JR (2014) A new efficient method for synaptic vesicle quantification reveals differences between medial prefrontal cortex perforated and nonperforated synapses. J Comp Neurol 522:284-297.
Ochsner KN, Ray RD, Cooper JC, Robertson ER, Chopra S, Gabrieli JD, Gross JJ (2004) For better or for worse: neural systems supporting the cognitive down- and up-regulation of negative emotion. Neuroimage 23:483-499.

Ongur D, An X, Price JL (1998) Prefrontal cortical projections to the hypothalamus in macaque monkeys. J Comp Neurol 401:480-505.

Opler LA, Opler MG, Arnsten AF (2016) Ameliorating treatment-refractory depression with intranasal ketamine: potential NMDA receptor actions in the pain circuitry representing mental anguish. CNS Spectr 21:12-22.

Palomero-Gallagher N, Mohlberg H, Zilles K, Vogt B (2008) Cytology and receptor architecture of human anterior cingulate cortex. J Comp Neurol 508:906-926.

Palomero-Gallagher N, Vogt BA, Schleicher A, Mayberg HS, Zilles K (2009) Receptor architecture of human cingulate cortex: evaluation of the fourregion neurobiological model. Hum Brain Mapp 30:2336-2355.

Peters A, Palay SL, Webster HD (1991) The fine structure of the nervous system. In: Neurons and their supporting cells. Oxford: Oxford UP.

Pizzagalli D, Pascual-Marqui RD, Nitschke JB, Oakes TR, Larson CL, Abercrombie HC, Schaefer SM, Koger JV, Benca RM, Davidson RJ (2001) Anterior cingulate activity as a predictor of degree of treatment response in major depression: evidence from brain electrical tomography analysis. Am J Psychiatry 158:405-415.

Ramirez-Mahaluf JP, Roxin A, Mayberg HS, Compte A (2017) A computational model of major depression: the role of glutamate dysfunction on cingulo-frontal network dynamics. Cereb Cortex 27:660-679.

Ramirez-Mahaluf JP, Perramon J, Otal B, Villoslada P, Compte A (2018) Subgenual anterior cingulate cortex controls sadness-induced modulations of cognitive and emotional network hubs. Sci Rep 8:8566.

Rasband WS (1997) ImageJ. Bethesda, MD: National Institutes of Health.

Reiner A, Veenman CL, Medina L, Jiao Y, Del Mar N, Honig MG (2000) Pathway tracing using biotinylated dextran amines. J Neurosci Methods 103:23-37.

Reinstatler L, Youssef NA (2015) Ketamine as a potential treatment for suicidal ideation: a systematic review of the literature. Drugs R D 15:37-43.

Rempel-Clower NL, Barbas H (1998) Topographic organization of connections between the hypothalamus and prefrontal cortex in the rhesus monkey. J Comp Neurol 398:393-419.

Richmond FJ, Gladdy R, Creasy JL, Kitamura S, Smits E, Thomson DB (1994) Efficacy of seven retrograde tracers, compared in multiple-labelling studies of feline motoneurones. J Neurosci Methods 53:35-46.

Riva-Posse P, Choi KS, Holtzheimer PE, McIntyre CC, Gross RE, Chaturvedi A, Crowell AL, Garlow SJ, Rajendra JK, Mayberg HS (2014) Defining critical white matter pathways mediating successful subcallosal cingulate deep brain stimulation for treatment-resistant depression. Biol Psychiatry 76:963-969.

Rive MM, van Rooijen G, Veltman DJ, Phillips ML, Schene AH, Ruhé HG (2013) Neural correlates of dysfunctional emotion regulation in major depressive disorder: a systematic review of neuroimaging studies. Neurosci Biobehav Rev 37:2529-2553.

Rollenhagen A, Lubke JH (2006) The morphology of excitatory central synapses: from structure to function. Cell Tissue Res 326:221-237.

Salvadore G, Cornwell BR, Colon-Rosario V, Coppola R, Grillon C, Zarate CA, Manji HK (2009) Increased anterior cingulate cortical activity in response to fearful faces: a neurophysiological biomarker that predicts rapid antidepressant response to ketamine. Biol Psychiatry 65:289-295.

Segal M, Vlachos A, Korkotian E (2010) The spine apparatus, synaptopodin, and dendritic spine plasticity. Neuroscientist 16:125-131.

Semendeferi K, Armstrong E, Schleicher A, Zilles K, Van Hoesen GW (2001) Prefrontal cortex in humans and apes: a comparative study of area 10. Am J Phys Anthropol 114:224-241.

Semendeferi K, Teffer K, Buxhoeveden DP, Park MS, Bludau S, Amunts K, Travis K, Buckwalter J (2011) Spatial organization of neurons in the frontal pole sets humans apart from great apes. Cereb Cortex 21:1485-1497.

Seminowicz DA, Mayberg HS, McIntosh AR, Goldapple K, Kennedy S, Segal Z, Rafi-Tari S (2004) Limbic-frontal circuitry in major depression: a path modeling metanalysis. Neuroimage 22:409-418.

Sinha R, Lacadie CM, Constable RT, Seo D (2016) Dynamic neural activity during stress signals resilient coping. Proc Natl Acad Sci USA 113:88378842 .

Stevens CF (2004) Systems biology versus molecular biology. Curr Biol 14: R51-R52. 
Tang W, Jbabdi S, Zhu Z, Cottaar M, Grisot G, Lehman JF, Yendiki A, Haber SN (2019) A connectional hub in the rostral anterior cingulate cortex links areas of emotion and cognitive control. eLife 8:e43761.

Telenczuk B, Dehghani N, Le Van Quyen M, Cash SS, Halgren E, Hatsopoulos NG, Destexhe A (2017) Local field potentials primarily reflect inhibitory neuron activity in human and monkey cortex. Sci Rep 7:40211.

Thomson AM (2000) Molecular frequency filters at central synapses. Prog Neurobiol 62:159-196.

Timbie C, Garcia-Cabezas MA, Zikopoulos B, Barbas H (2020) Organization of primate amygdalar-thalamic pathways for emotions. PLoS Biol 18: e3000639.

Trevizol AP, Blumberger DM (2019) An update on repetitive transcranial magnetic stimulation for the treatment of major depressive disorder. Clin Pharmacol Ther 106:747-762.

Veenman CL, Reiner A, Honig MG (1992) Biotinylated dextran amine as an anterograde tracer for single- and double-labeling studies. J Neurosci Methods 41:239-254.

Wallis CU, Cardinal RN, Alexander L, Roberts AC, Clarke HF (2017) Opposing roles of primate areas 25 and 32 and their putative rodent homologs in the regulation of negative emotion. Proc Natl Acad Sci USA 114:E4075-E4084.

Wang M, Yang Y, Wang CJ, Gamo NJ, Jin LE, Mazer JA, Morrison JH, Wang XJ, Arnsten AF (2013) NMDA receptors subserve persistent neuronal firing during working memory in dorsolateral prefrontal cortex. Neuron 77:736-749.

Wang XJ, Tegner J, Constantinidis C, Goldman-Rakic PS (2004) Division of labor among distinct subtypes of inhibitory neurons in a cortical microcircuit of working memory. Proc Natl Acad Sci USA 101:1368-1373.
Wang YL, Yang SZ, Sun WL, Shi YZ, Duan HF (2016) Altered functional interaction hub between affective network and cognitive control network in patients with major depressive disorder. Behav Brain Res 298:301-309.

Waters AC, Mayberg HS (2017) Brain-based biomarkers for the treatment of depression: evolution of an idea. J Int Neuropsychol Soc 23:870-880

Weigand A, Horn A, Caballero R, Cooke D, Stern AP, Taylor SF, Press D, Pascual-Leone A, Fox MD (2018) Prospective validation that subgenual connectivity predicts antidepressant efficacy of transcranial magnetic stimulation sites. Biol Psychiatry 84:28-37.

Whitfield-Gabrieli S, Ford JM (2012) Default mode network activity and connectivity in psychopathology. Annu Rev Clin Psychol 8:49-76.

Zaitsev AV, Povysheva NV, Gonzalez-Burgos G, Rotaru D, Fish KN, Krimer LS, Lewis DA (2009) Interneuron diversity in layers 2-3 of monkey prefrontal cortex. Cereb Cortex 19:1597-1615.

Zeredo JL, Quah SK, Wallis CU, Alexander L, Cockcroft GJ, Santangelo AM, Xia J, Shiba Y, Dalley JW, Cardinal RN, Roberts AC, Clarke HF (2019) Glutamate within the marmoset anterior hippocampus interacts with area 25 to regulate the behavioral and cardiovascular correlates of hightrait anxiety. J Neurosci 39:3094-3107.

Zikopoulos B, Barbas H (2006) Prefrontal projections to the thalamic reticular nucleus form a unique circuit for attentional mechanisms. J Neurosci 26:7348-7361

Zikopoulos B, Garcia-Cabezas MA, Barbas H (2018) Parallel trends in cortical grey and white matter architecture and connections in primates allow fine study of pathways in humans and reveal network disruptions in autism. PLoS Biol 16:e2004559.

Zikopoulos B, John YJ, García-Cabezas MA, Bunce JG, Barbas H (2016) The intercalated nuclear complex of the primate amygdala. Neuroscience 330:267-290. 